Supporting Information for

\title{
Synthesis of Cationic Molybdenum Imido 2-Adamantylidene Complexes from Bispyrrolides via Cationic Pyrrolenine Complexes
}

\author{
Bhaskar Paul, Richard R. Schrock,* and Charlene Tsay \\ Department of Chemistry, University of California at Riverside, Riverside, California 92521 \\ Email: richard.schrock@ucr.edu
}

\section{Contents}

1. NMR Spectra of the Compounds .........................................................................S2-S14

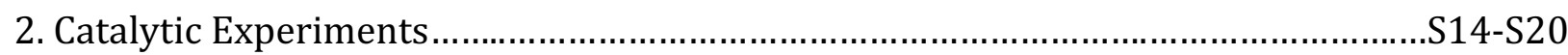

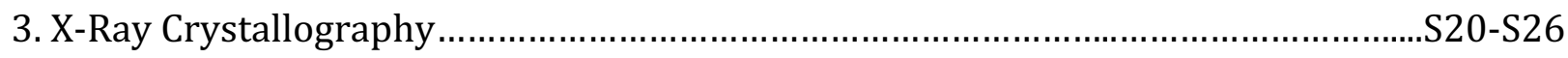

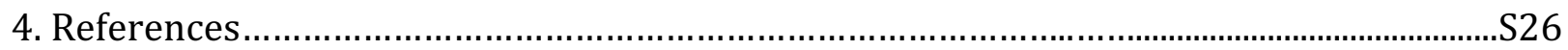




\section{NMR Spectra of Compounds}

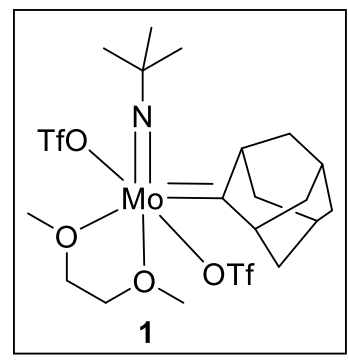

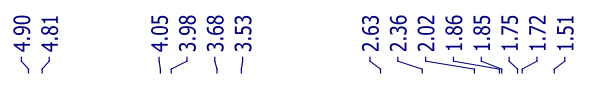
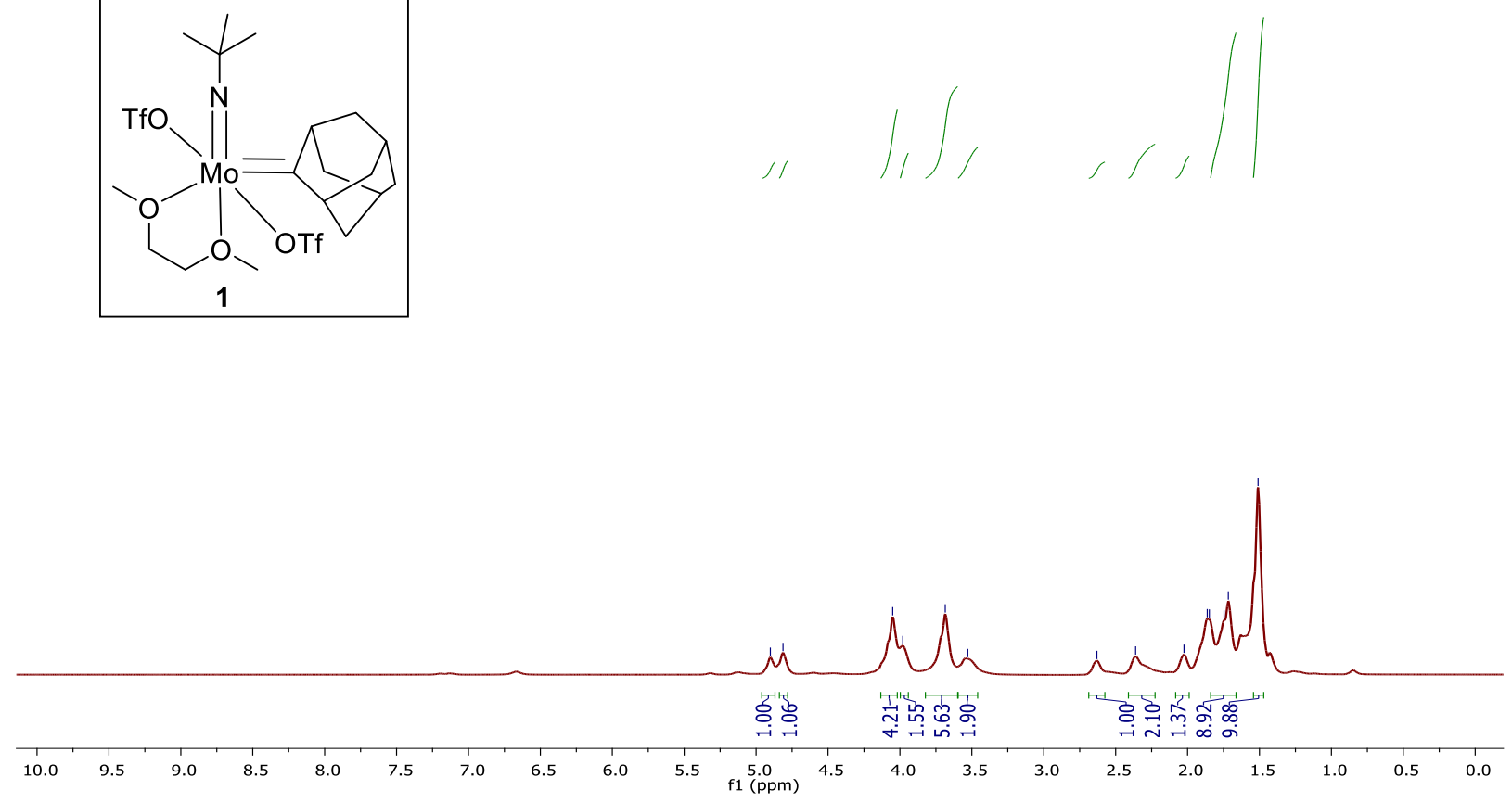

Figure S1. ${ }^{1} \mathrm{H}$ NMR spectrum of $\mathrm{Mo}(\mathrm{N}-t \text {-Bu)(Adene)(OTf) })_{2}(\mathrm{DME})(1)$ recorded at $600 \mathrm{MHz}$ in $\mathrm{CD}_{2} \mathrm{Cl}_{2}$.

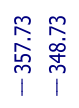

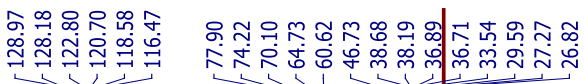

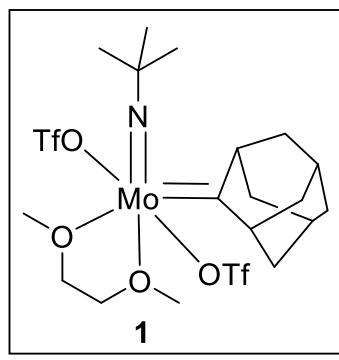

ulliti iो। 1 14:
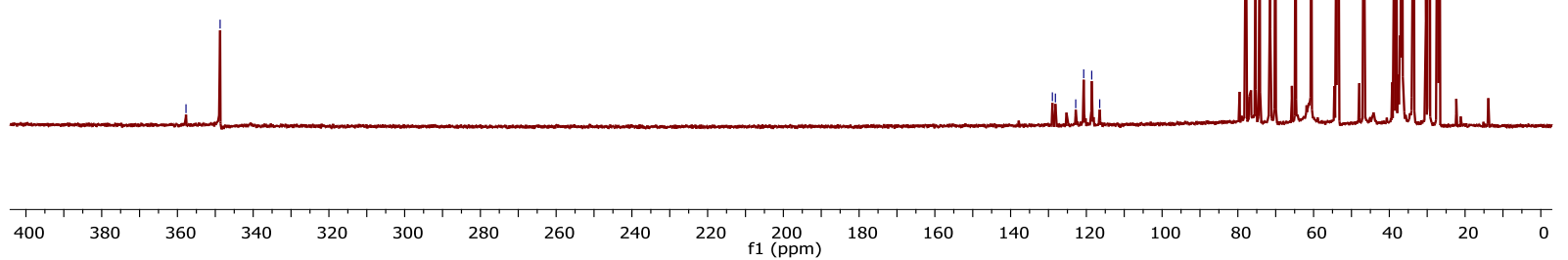

Figure S2. ${ }^{13} \mathrm{C}\left\{{ }^{1} \mathrm{H}\right\}$ NMR spectrum of $\mathrm{Mo}(\mathrm{N}-t-\mathrm{Bu})($ Adene)(OTf) $(\mathrm{DME})(\mathbf{1})$ recorded at $151 \mathrm{MHz}$ in $\mathrm{CD}_{2} \mathrm{Cl}_{2}$. 


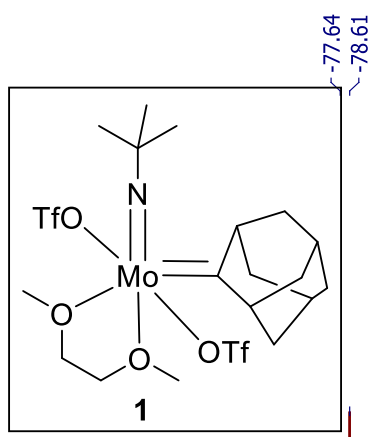

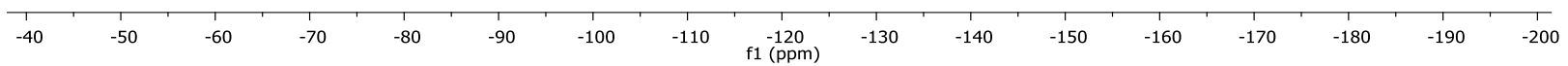

Figure S3. ${ }^{19} \mathrm{~F}\left\{{ }^{1} \mathrm{H}\right\}$ NMR spectrum of Mo(N-t-Bu)(Adene)(OTf) $)_{2}(\mathrm{DME})(\mathbf{1})$ recorded at $564 \mathrm{MHz}$ in $\mathrm{CD}_{2} \mathrm{Cl}_{2}$.

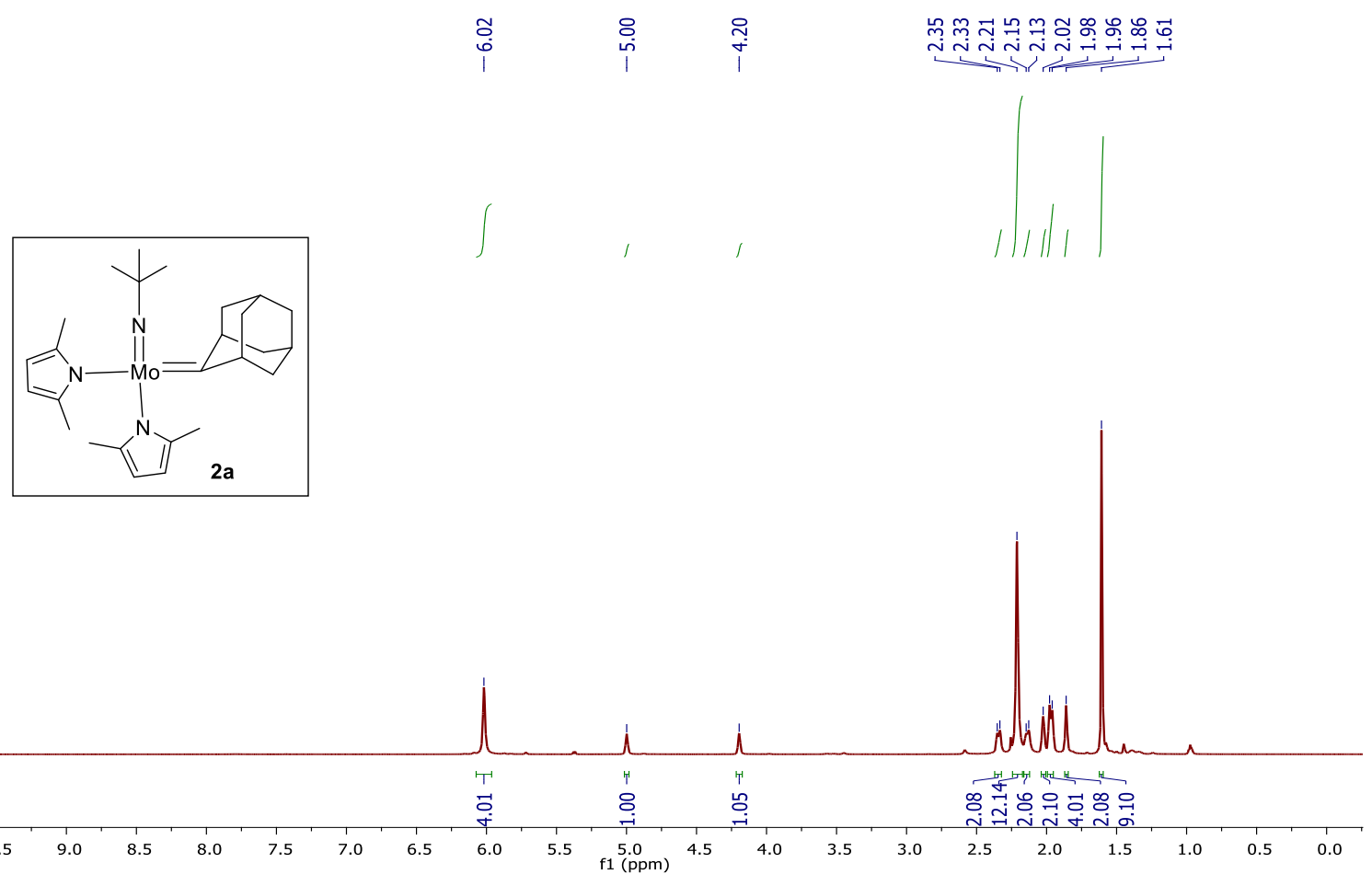

Figure S4. ${ }^{1} \mathrm{H}$ NMR spectrum of $\mathrm{Mo}\left(\mathrm{N}-t\right.$-Bu)(Adene) $\left(\mathrm{Me}_{2} \mathrm{Pyr}\right)_{2}(2 \mathrm{a})$ recorded at $600 \mathrm{MHz}$ in $\mathrm{CD}_{2} \mathrm{Cl}_{2}$. 

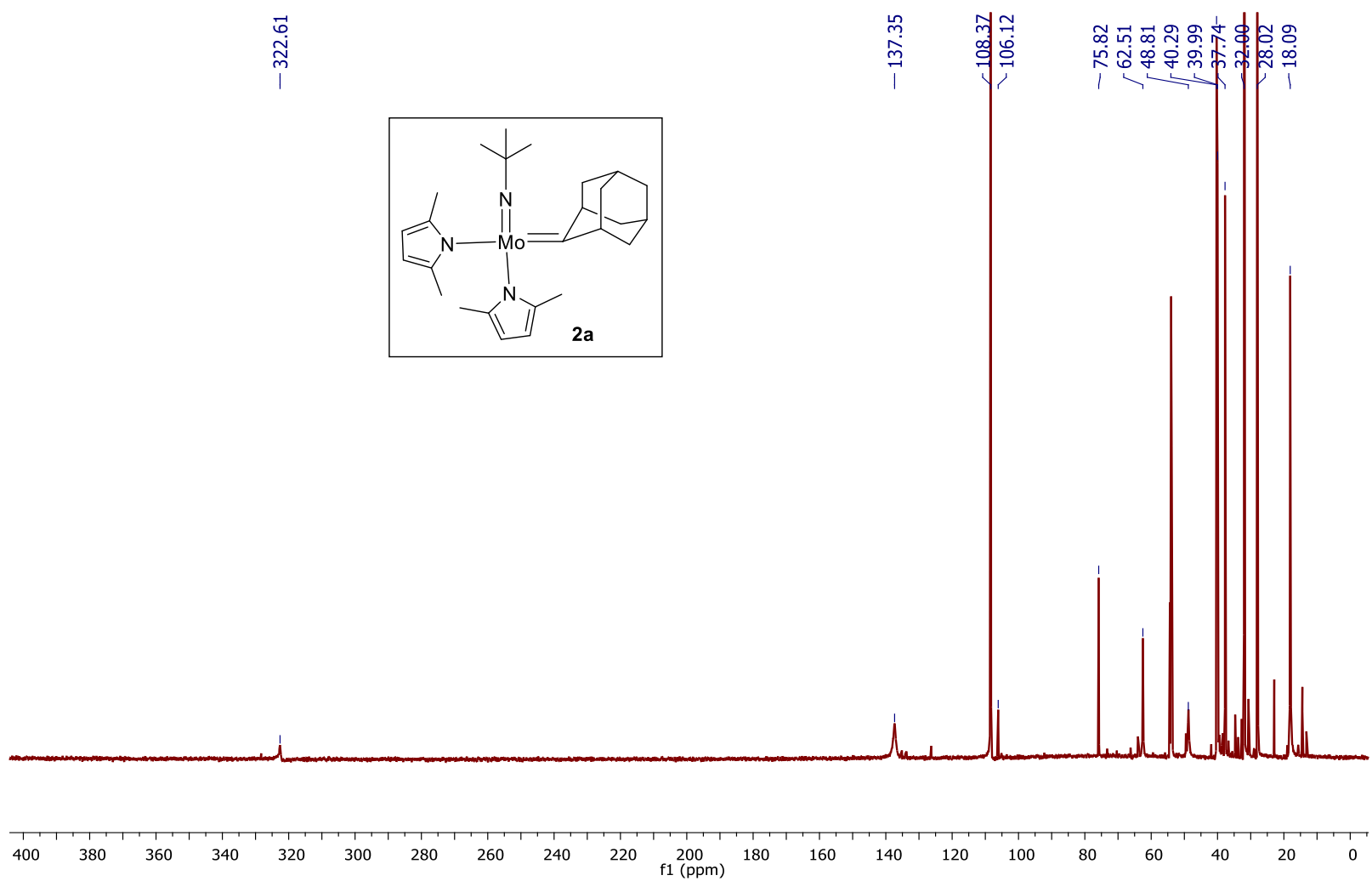

Figure S5. ${ }^{13} \mathrm{C}\left\{{ }^{1} \mathrm{H}\right\}$ NMR spectrum of $\mathrm{Mo}\left(\mathrm{N}-t \text {-Bu)(Adene)( } \mathrm{Me}_{2} \mathrm{Pyr}\right)_{2}$ (2a) recorded at $151 \mathrm{MHz}$ in $\mathrm{CD}_{2} \mathrm{Cl}_{2}$.

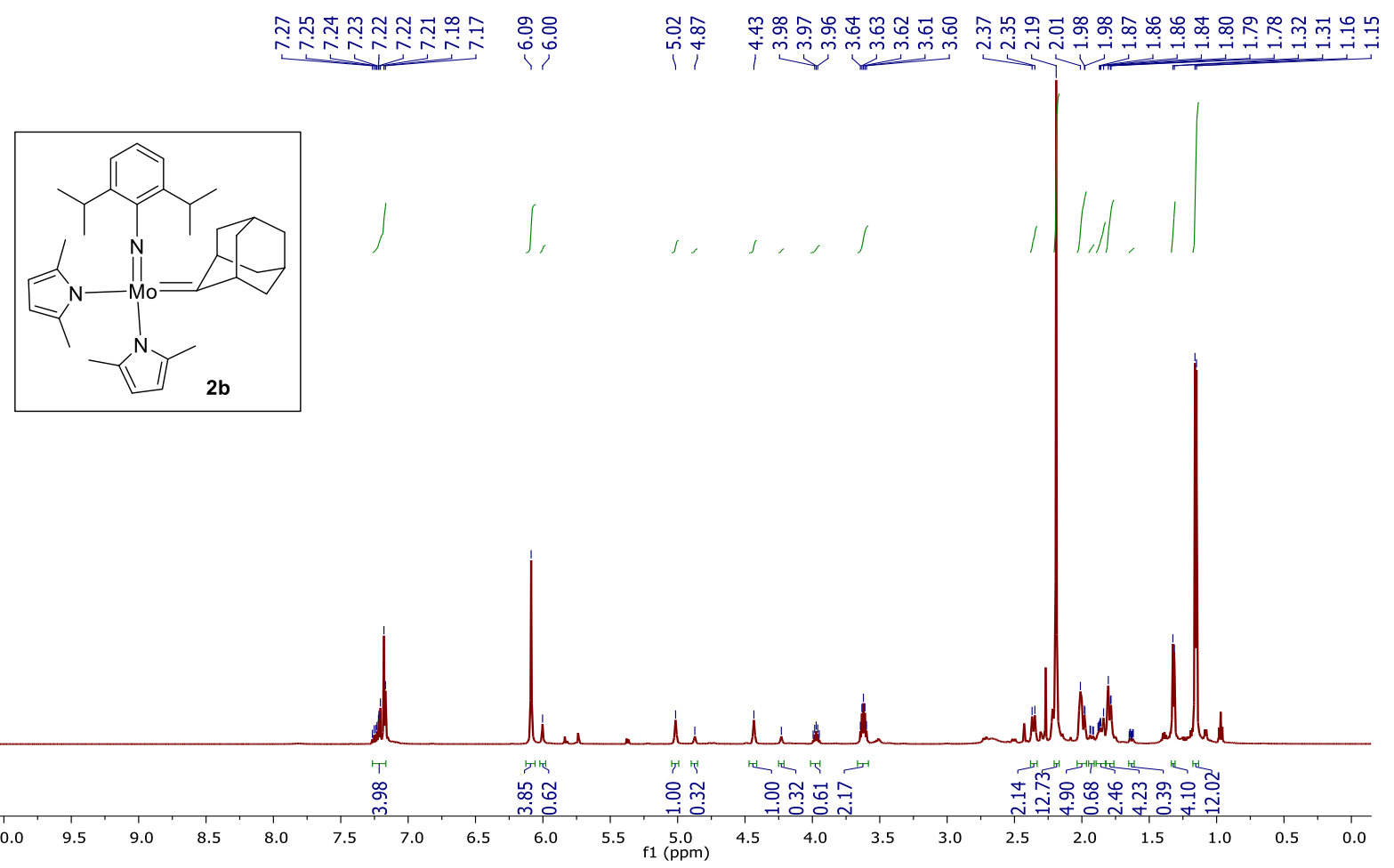

Figure S6. ${ }^{1} \mathrm{H}$ NMR spectrum of $\mathrm{Mo}(\mathrm{NAr})(A d e n e)\left(\mathrm{Me}_{2} \mathrm{Pyr}\right)_{2}(\mathbf{2 b})$ recorded at $600 \mathrm{MHz}$ in $\mathrm{CD}_{2} \mathrm{Cl}_{2}$. 

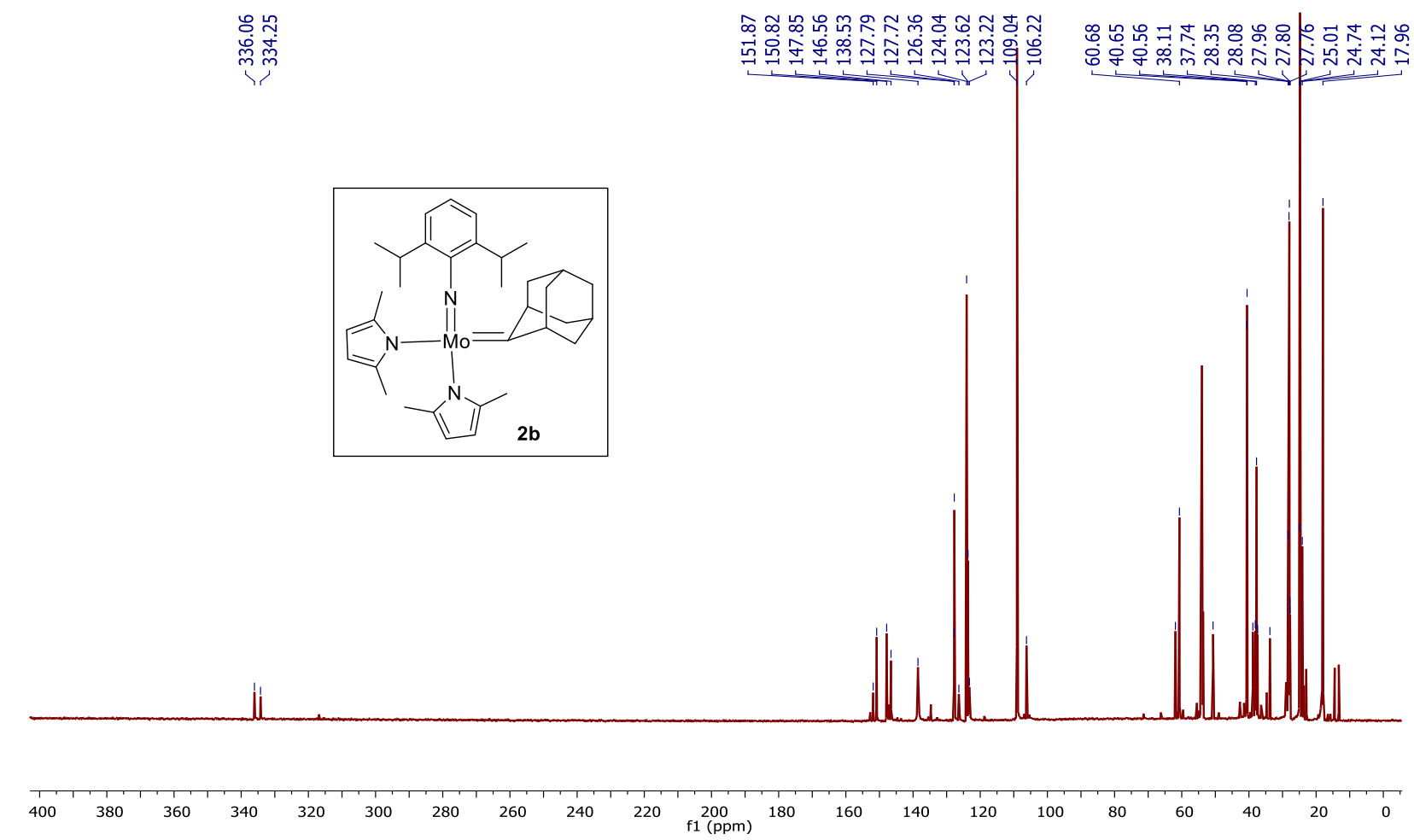

Figure S7. ${ }^{13} \mathrm{C}\left\{{ }^{1} \mathrm{H}\right\}$ NMR spectrum of Mo(NAr)(Adene) $\left(\mathrm{Me}_{2} \mathrm{Pyr}\right)_{2}(\mathbf{2 b})$ recorded at $151 \mathrm{MHz}$ in $\mathrm{CD}_{2} \mathrm{Cl}_{2}$.

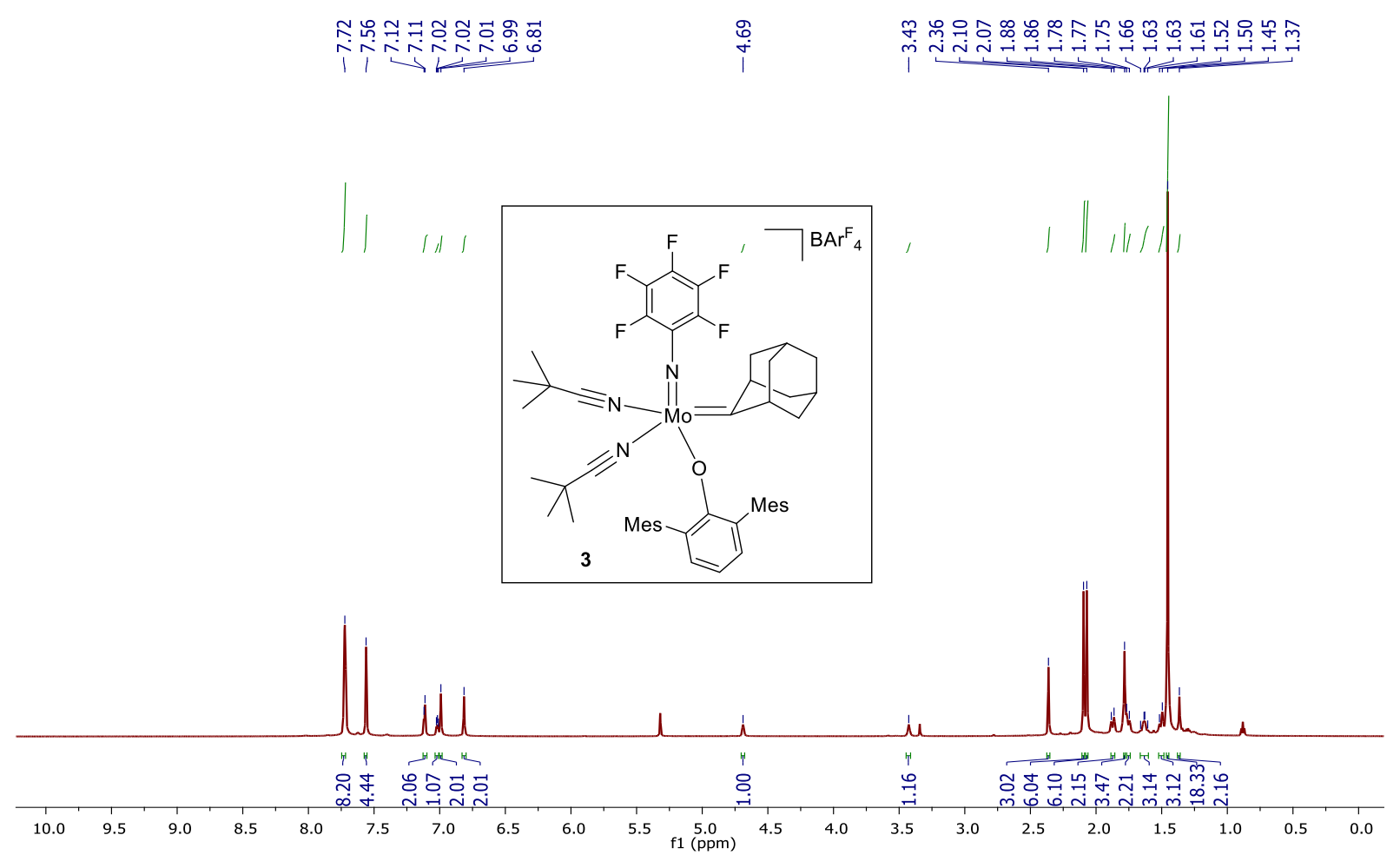

Figure S8. ${ }^{1} \mathrm{H}$ NMR spectrum of $\mathrm{Mo}\left(\mathrm{NC}_{6} \mathrm{~F}_{5}\right)\left(\right.$ Adene)(OHMT)(Piv) ${ }_{2}\left(\mathrm{BAr}^{\mathrm{F}} 4\right)(3)$ recorded at $600 \mathrm{MHz}$ in $\mathrm{CD}_{2} \mathrm{Cl}_{2}$. 


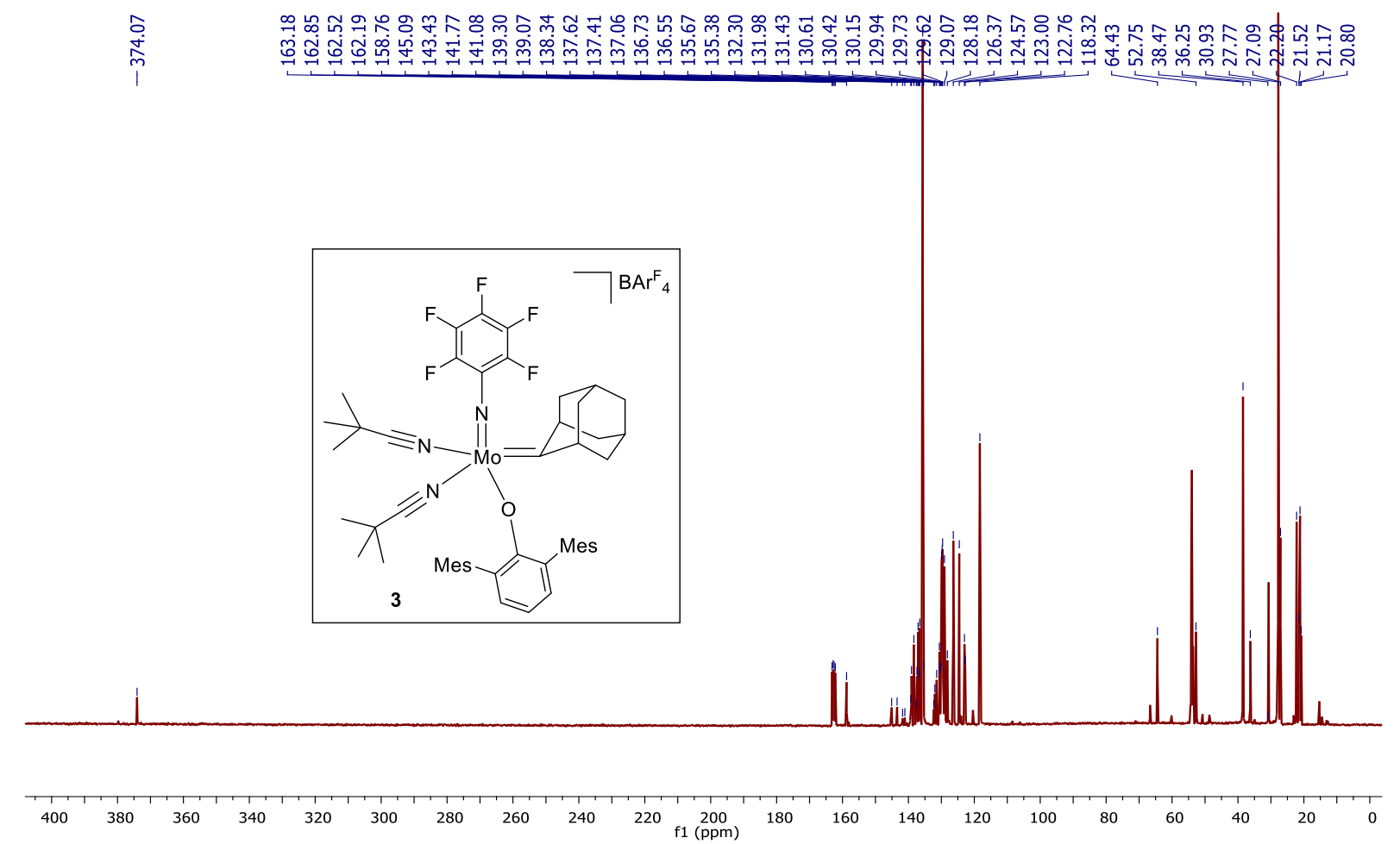

Figure S9. ${ }^{13} \mathrm{C}\left\{{ }^{1} \mathrm{H}\right\}$ NMR spectrum of $\mathrm{Mo}\left(\mathrm{NC}_{6} \mathrm{~F}_{5}\right)(\text { Adene)(OHMT)(Piv) })_{2}\left(\mathrm{BAr}^{\mathrm{F}} 4\right)(3)$ recorded at 151 $\mathrm{MHz}$ in $\mathrm{CD}_{2} \mathrm{Cl}_{2}$.
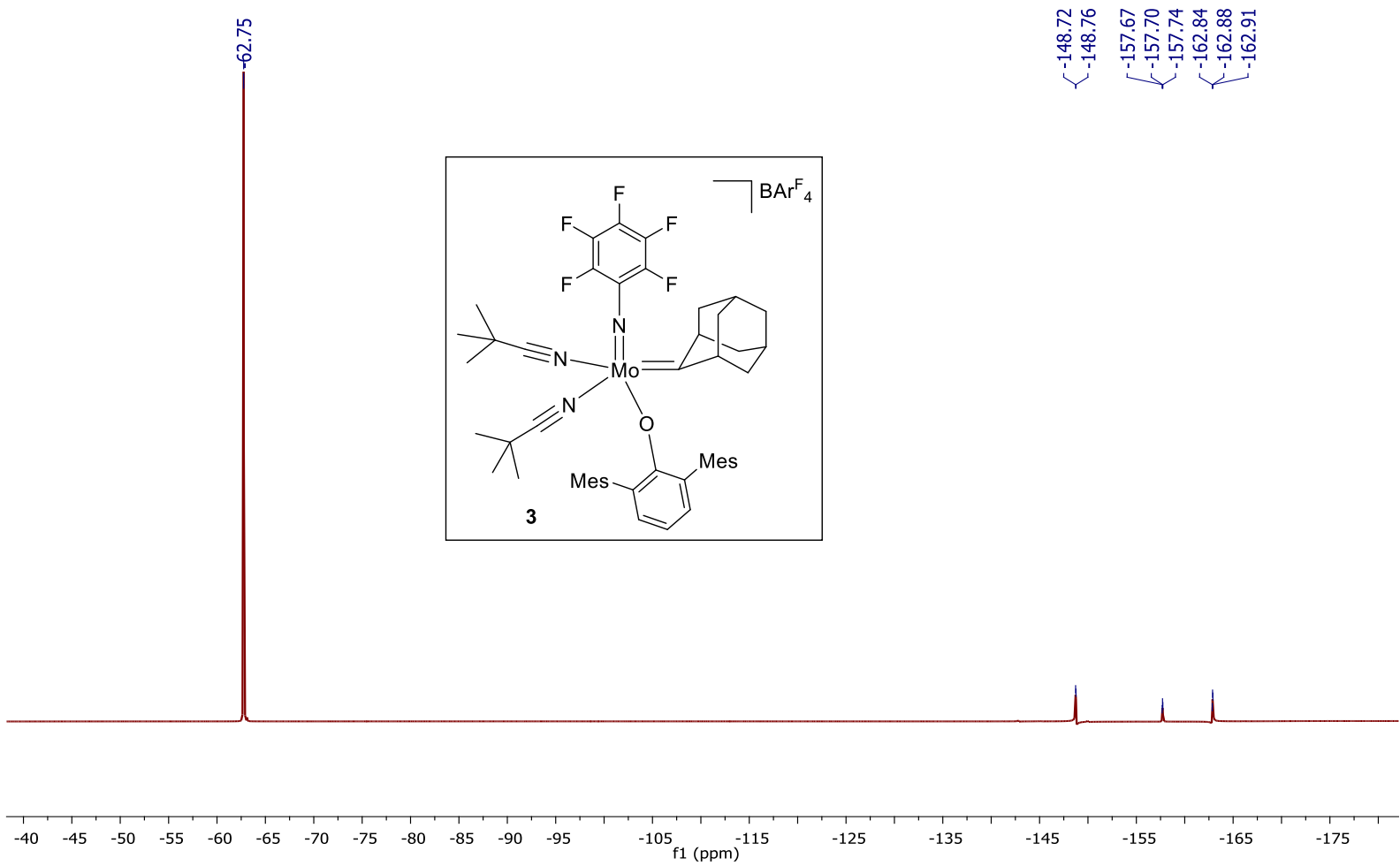

Figure S10. ${ }^{19} \mathrm{~F}\left\{{ }_{1} \mathrm{H}\right\}$ NMR spectrum of $\mathrm{Mo}\left(\mathrm{NC}_{6} \mathrm{~F}_{5}\right)$ (Adene)(OHMT)(Piv) ${ }_{2}\left(\mathrm{BAr}_{4}{ }_{4}\right)(3)$ recorded at 564 $\mathrm{MHz}$ in $\mathrm{CD}_{2} \mathrm{Cl}_{2}$. 


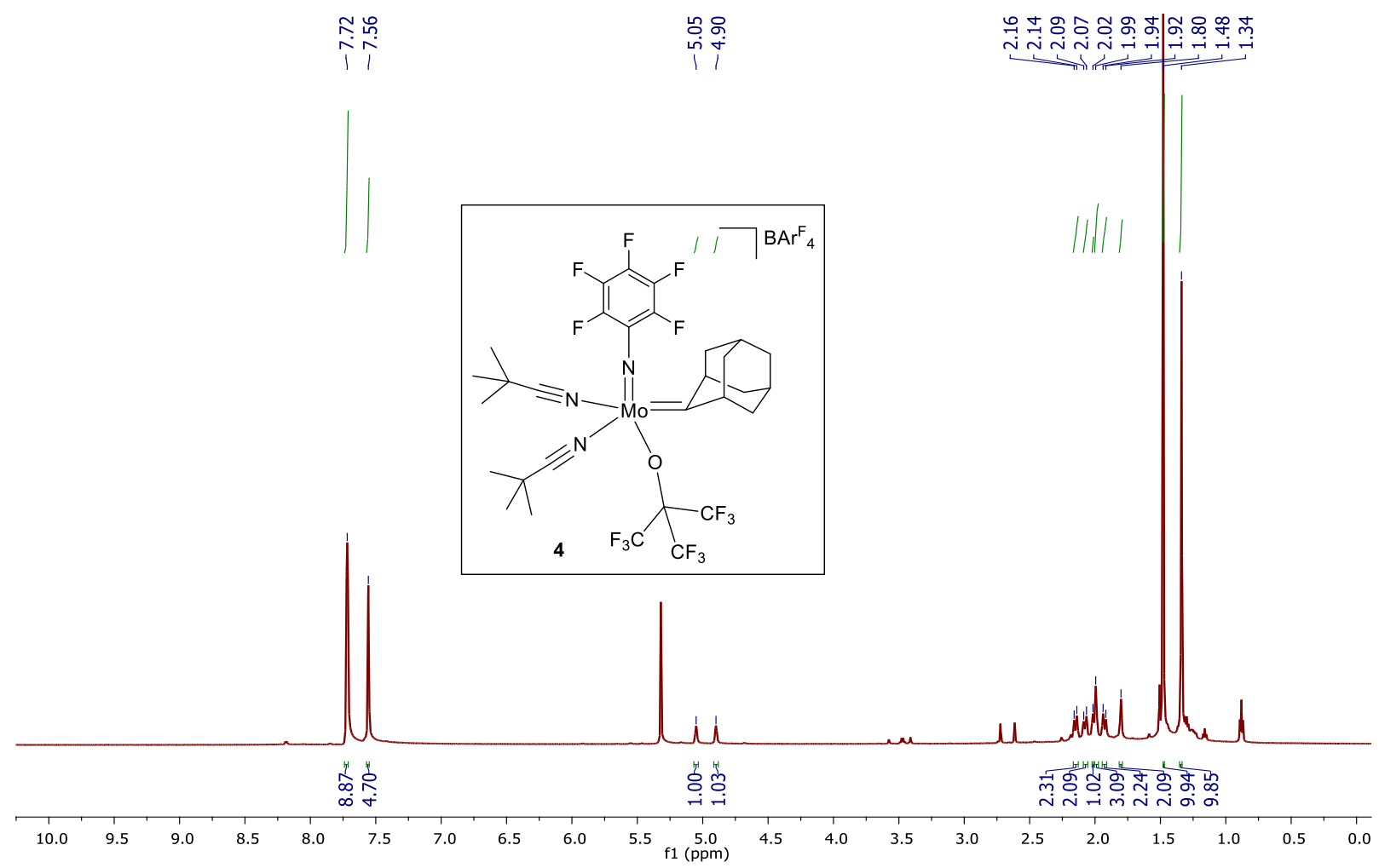

Figure S11. ${ }^{1} \mathrm{H}$ NMR spectrum of $\mathrm{Mo}\left(\mathrm{NC}_{6} \mathrm{~F}_{5}\right)\left(\right.$ Adene) $\left(\mathrm{OC}_{4} \mathrm{~F}_{9}\right)\left(\mathrm{Piv}_{2}\left(\mathrm{BAr}_{4}\right)(4)\right.$ recorded at $600 \mathrm{MHz}$ in $\mathrm{CD}_{2} \mathrm{Cl}_{2}$.

芯

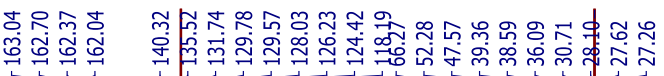

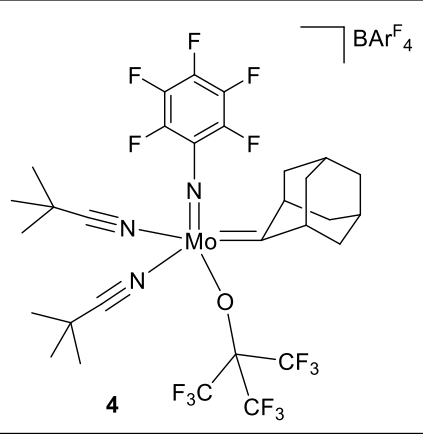

1 iudtid doldon.
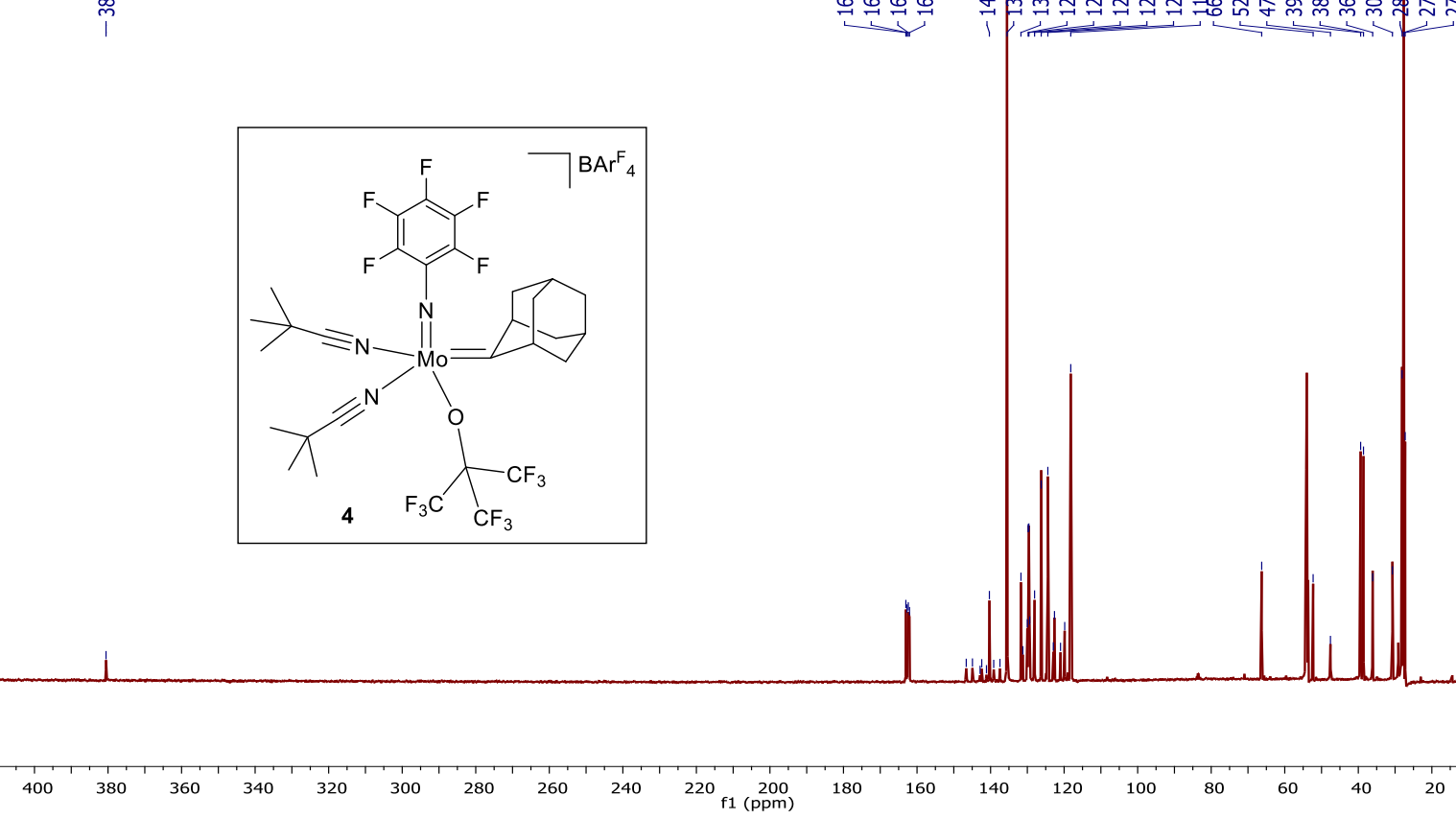

Figure S12. ${ }^{13} \mathrm{C}\left\{{ }^{1} \mathrm{H}\right\}$ NMR spectrum of $\mathrm{Mo}\left(\mathrm{NC}_{6} \mathrm{~F}_{5}\right)($ Adene $)\left(\mathrm{OC}_{4} \mathrm{~F}_{9}\right)(\mathrm{Piv})_{2}\left(\mathrm{BAr}_{4}{ }^{\mathrm{F}}\right)(4)$ recorded at 151 $\mathrm{MHz}$ in $\mathrm{CD}_{2} \mathrm{Cl}_{2}$. 

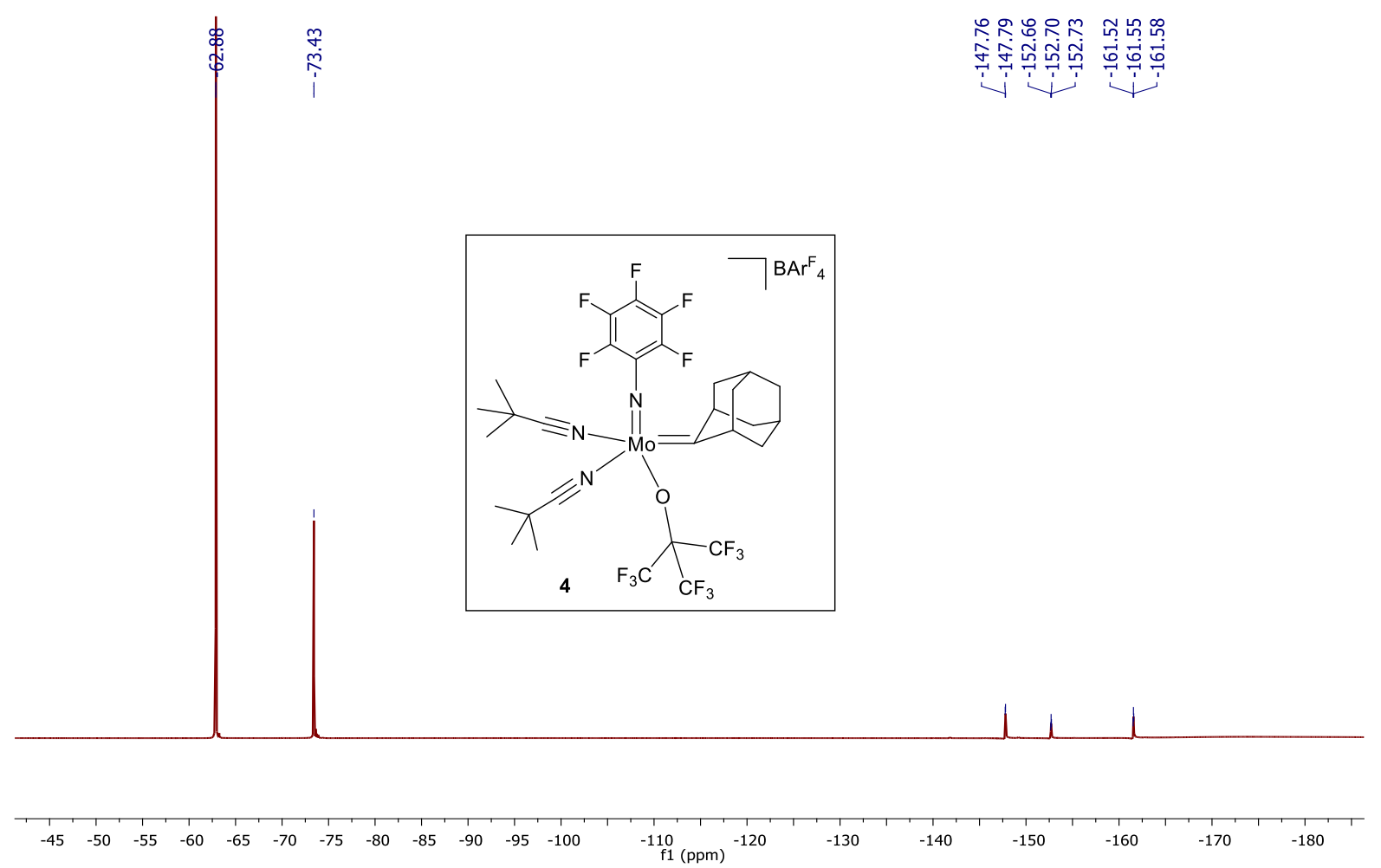

Figure S13. ${ }^{9} \mathrm{~F}\left\{{ }_{1} \mathrm{H}\right\}$ NMR spectrum of $\mathrm{Mo}\left(\mathrm{NC}_{6} \mathrm{~F}_{5}\right)($ Adene $)\left(\mathrm{OC}_{4} \mathrm{~F}_{9}\right)\left(\mathrm{Piv}_{2}\left(\mathrm{BAr}_{4}\right)(4)\right.$ recorded at 564 $\mathrm{MHz}$ in $\mathrm{CD}_{2} \mathrm{Cl}_{2}$.

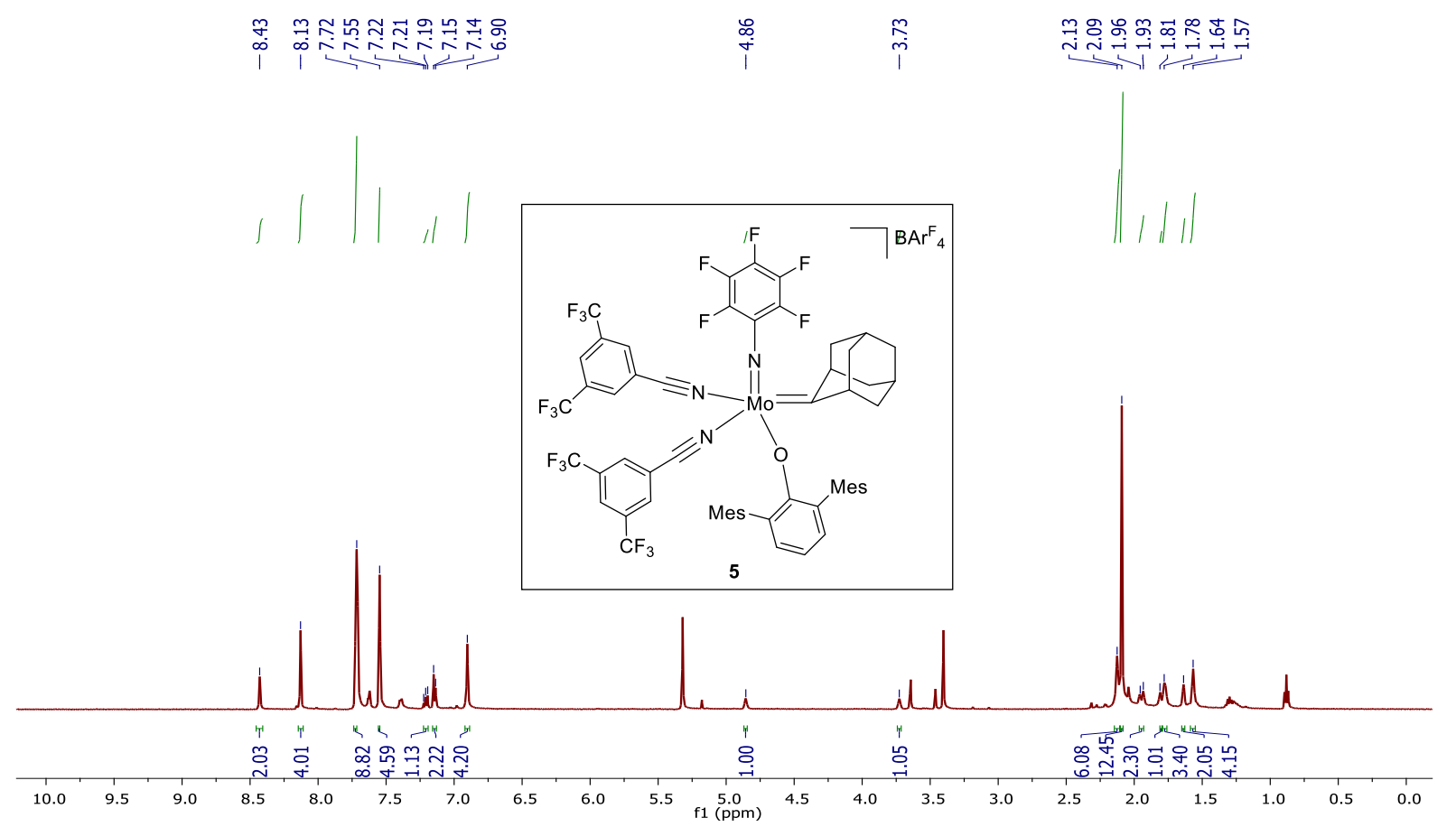

Figure S14. ${ }^{1} \mathrm{H}$ NMR spectrum of $\mathrm{Mo}\left(\mathrm{NC}_{6} \mathrm{~F}_{5}\right)($ Adene $)(\mathrm{OHMT})\left(\mathrm{Ph}^{\mathrm{F} 6} \mathrm{CN}\right)_{2}\left(\mathrm{BAr}_{4}\right)(5)$ recorded at 600 $\mathrm{MHz}$ in $\mathrm{CD}_{2} \mathrm{Cl}_{2}$. 


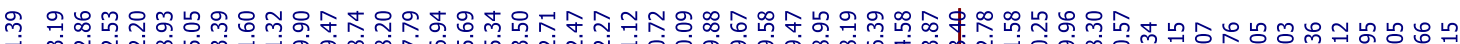

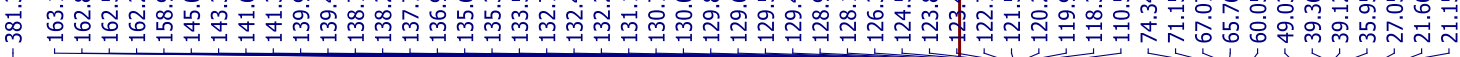

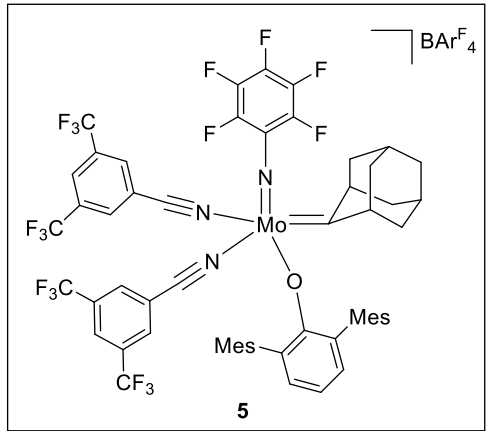

5
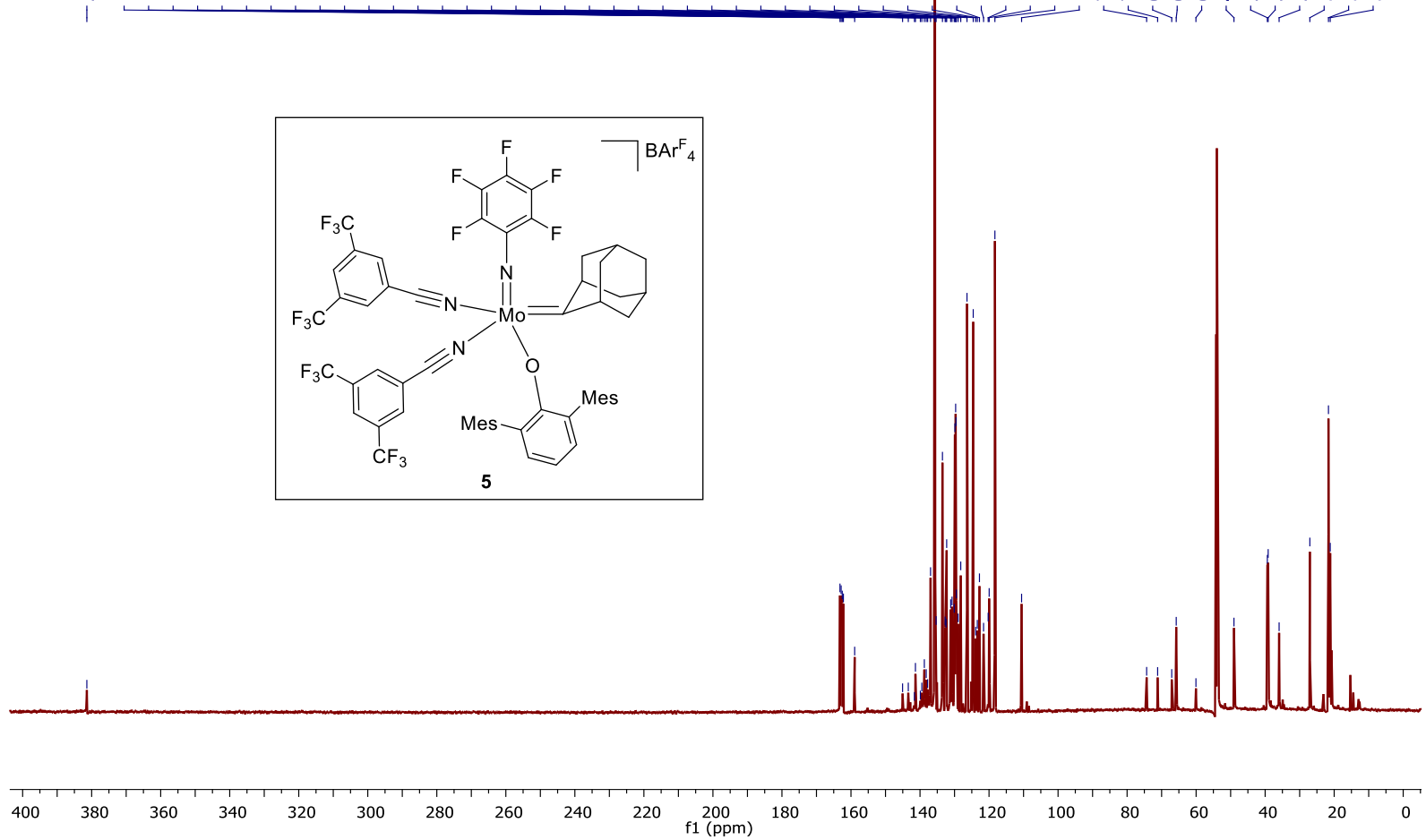

Figure S15. ${ }^{13} \mathrm{C}\left\{{ }^{1} \mathrm{H}\right\} \mathrm{NMR}$ spectrum of $\mathrm{Mo}\left(\mathrm{NC}_{6} \mathrm{~F}_{5}\right)($ Adene $)(\mathrm{OHMT})\left(\mathrm{Ph}^{\mathrm{F} 6} \mathrm{CN}\right)_{2}\left(\mathrm{BAr}_{4}\right)(5)$ recorded at $151 \mathrm{MHz}$ in $\mathrm{CD}_{2} \mathrm{Cl}_{2}$.
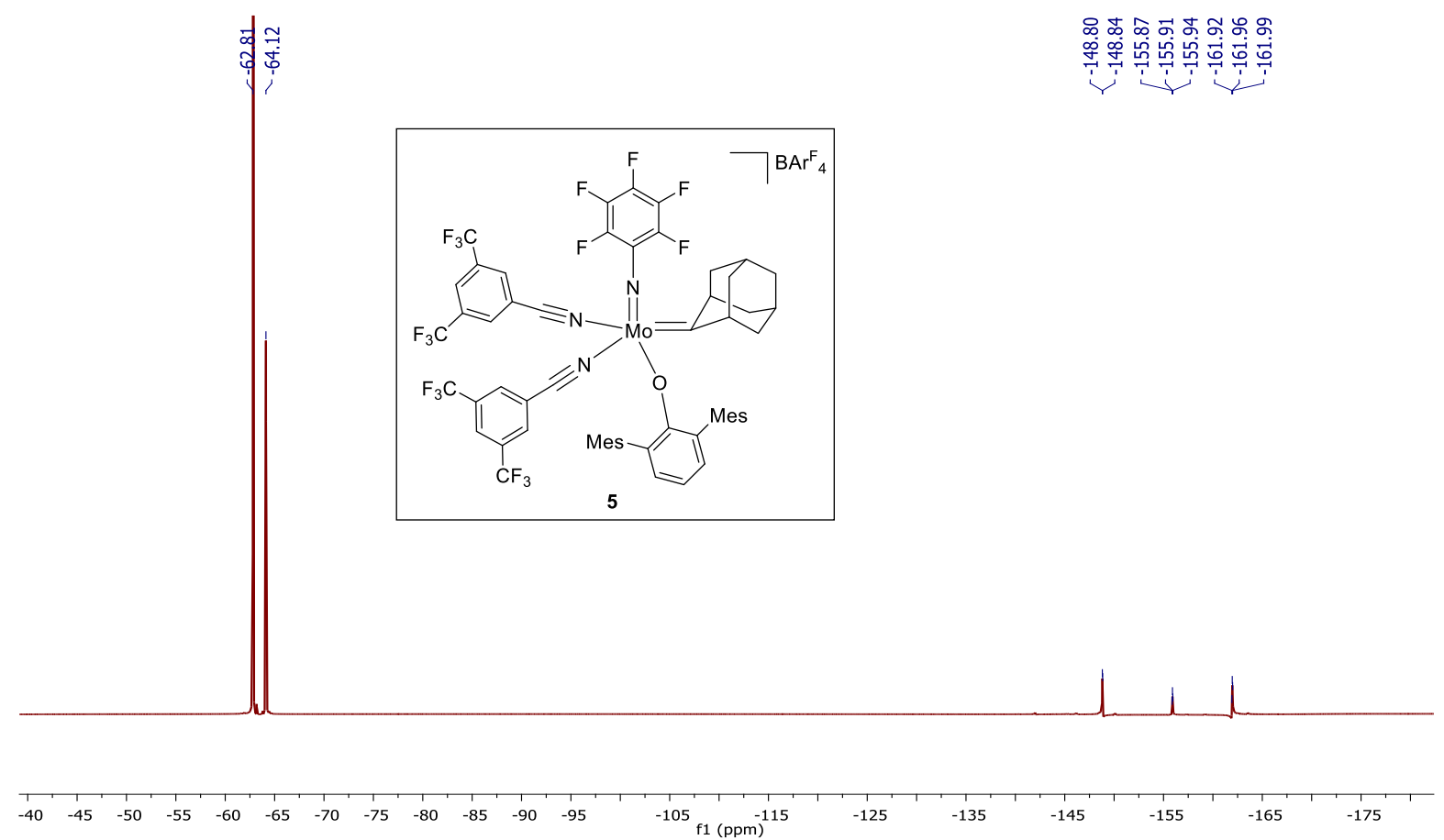

Figure S16. ${ }^{19} \mathrm{~F}\left\{{ }_{1} \mathrm{H}\right\}$ NMR spectrum of $\mathrm{Mo}\left(\mathrm{NC}_{6} \mathrm{~F}_{5}\right)($ Adene $)(\mathrm{OHMT})\left(\mathrm{Ph}^{\mathrm{F} 6} \mathrm{CN}\right)_{2}\left(\mathrm{BAr}_{4}\right)(5)$ recorded at $564 \mathrm{MHz}$ in $\mathrm{CD}_{2} \mathrm{Cl}_{2}$. 


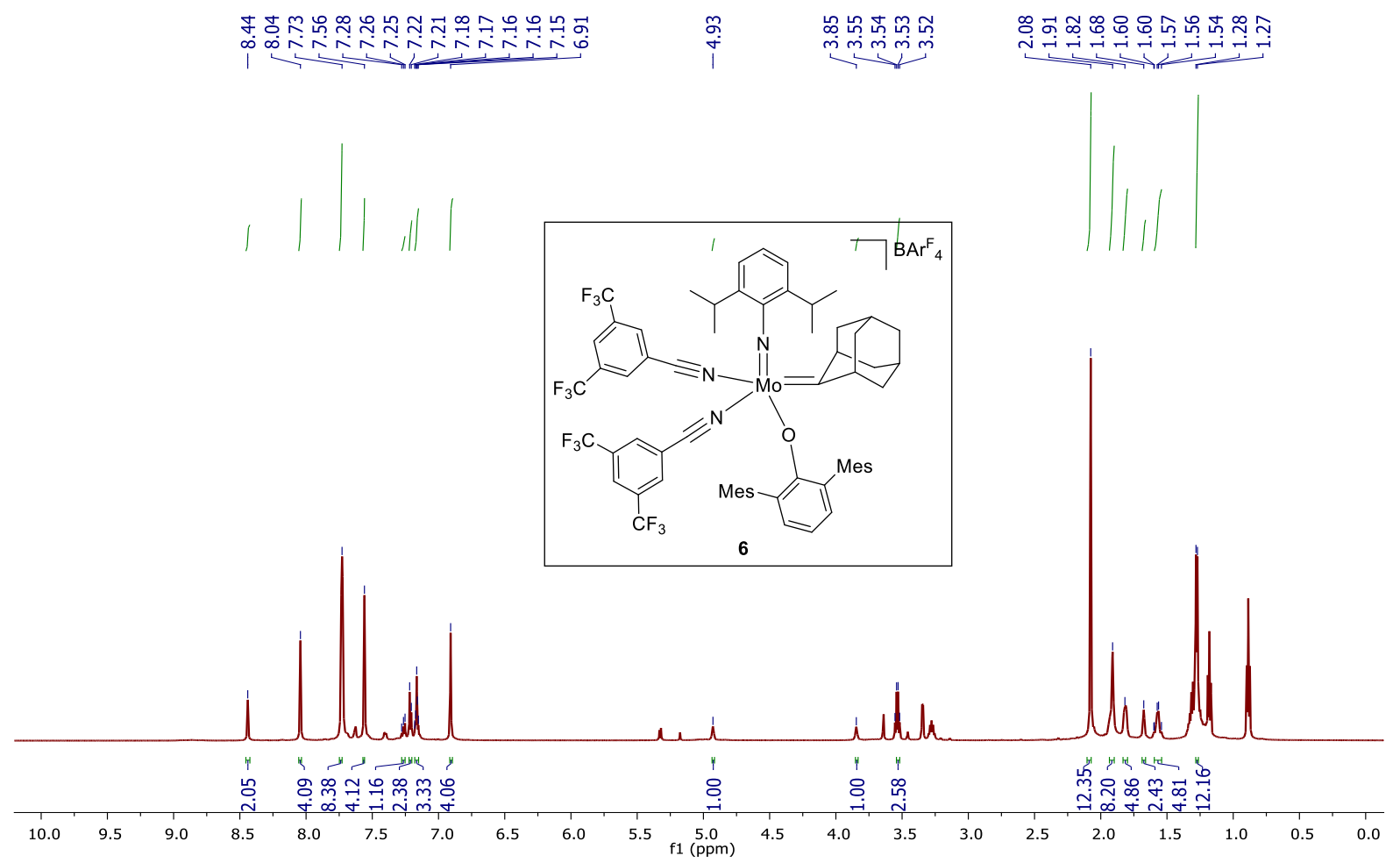

Figure S17. ${ }^{1} \mathrm{H}$ NMR spectrum of $\mathrm{Mo}(\mathrm{NAr})\left(\right.$ Adene) $(\mathrm{OHMT})\left(\mathrm{Ph}^{\mathrm{F} 6 \mathrm{CN}}\right)_{2}\left(\mathrm{BAr}_{4}{ }_{4}\right)(6)$ recorded at 600 $\mathrm{MHz}$ in $\mathrm{CD}_{2} \mathrm{Cl}_{2}$.

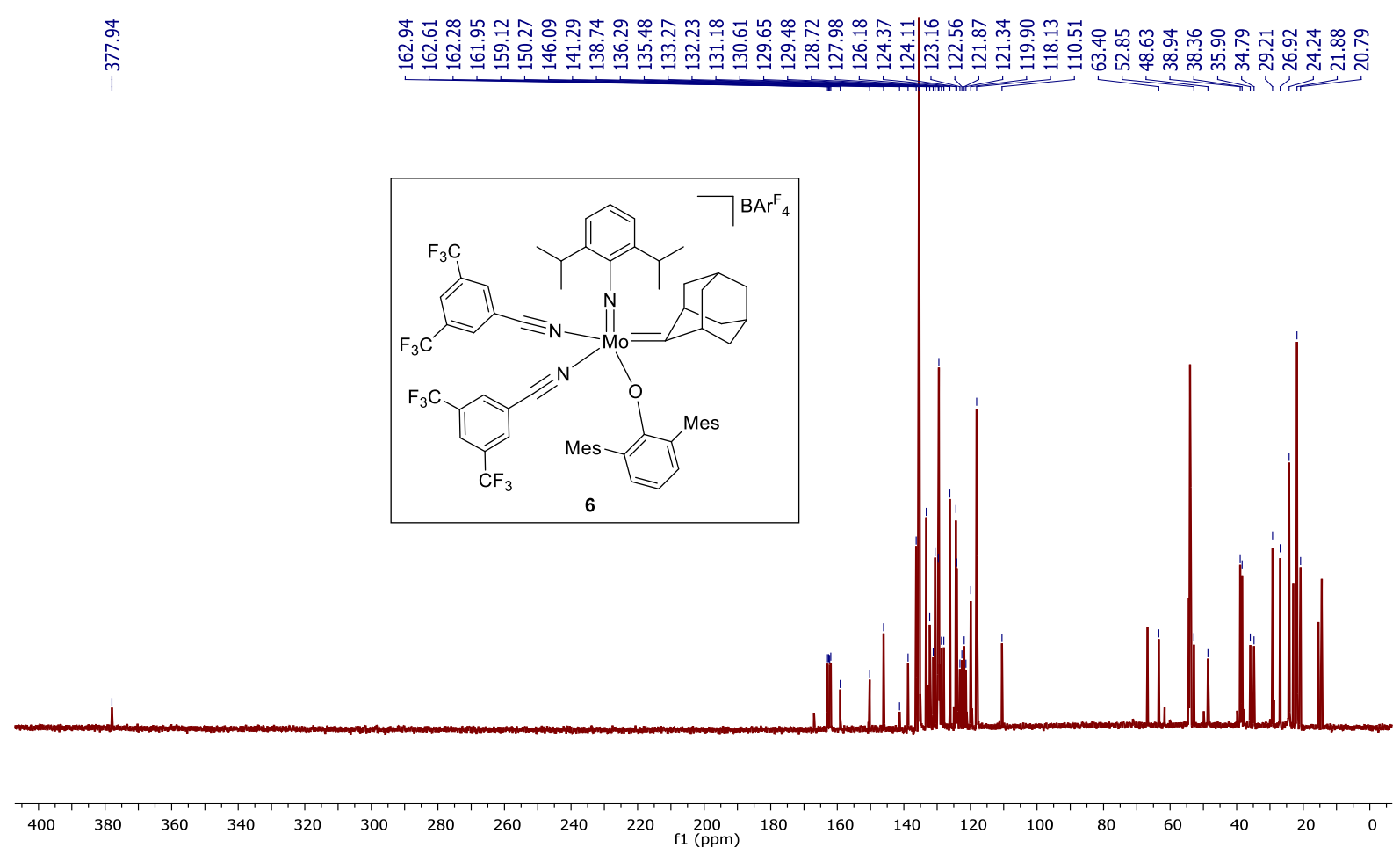

Figure S18. ${ }^{13} \mathrm{C}\left\{{ }^{1} \mathrm{H}\right\}$ NMR spectrum of $\mathrm{Mo}(\mathrm{NAr})\left(\right.$ Adene)(OHMT) $\left(\mathrm{Ph}^{\mathrm{F} 6} \mathrm{CN}\right)_{2}\left(\mathrm{BAr}_{4}{ }_{4}\right)(6)$ recorded at $151 \mathrm{MHz}$ in $\mathrm{CD}_{2} \mathrm{Cl}_{2}$. 

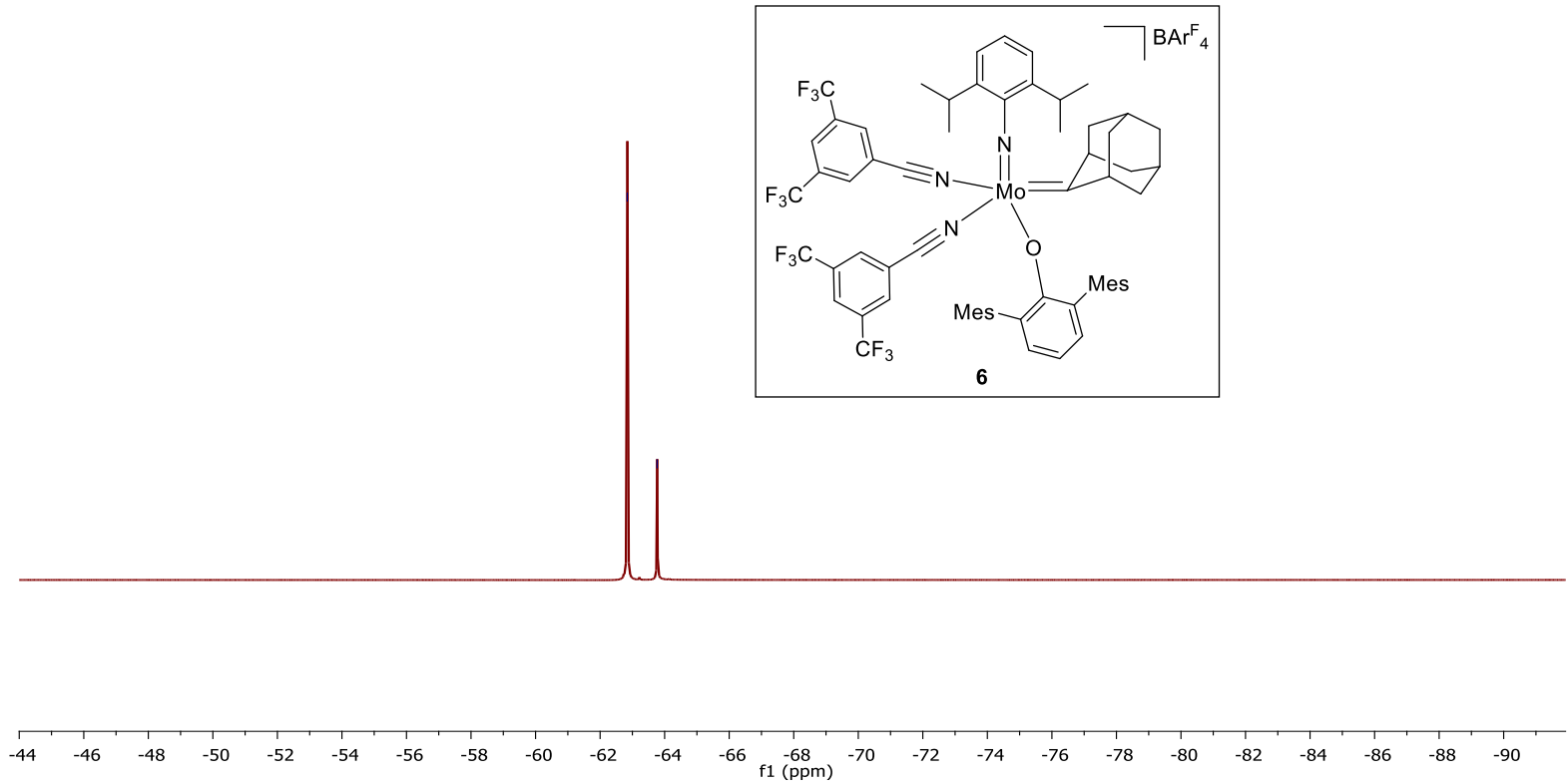

Figure S19. ${ }^{19} \mathrm{~F}\left\{{ }^{1} \mathrm{H}\right\}$ NMR spectrum of $\mathrm{Mo}(\mathrm{NAr})\left(\right.$ Adene) $(\mathrm{OHMT})\left(\mathrm{Ph}^{\mathrm{F} 6} \mathrm{CN}\right)_{2}\left(\mathrm{BAr}_{4}\right)(6)$ recorded at $564 \mathrm{MHz}$ in $\mathrm{CD}_{2} \mathrm{Cl}_{2}$.

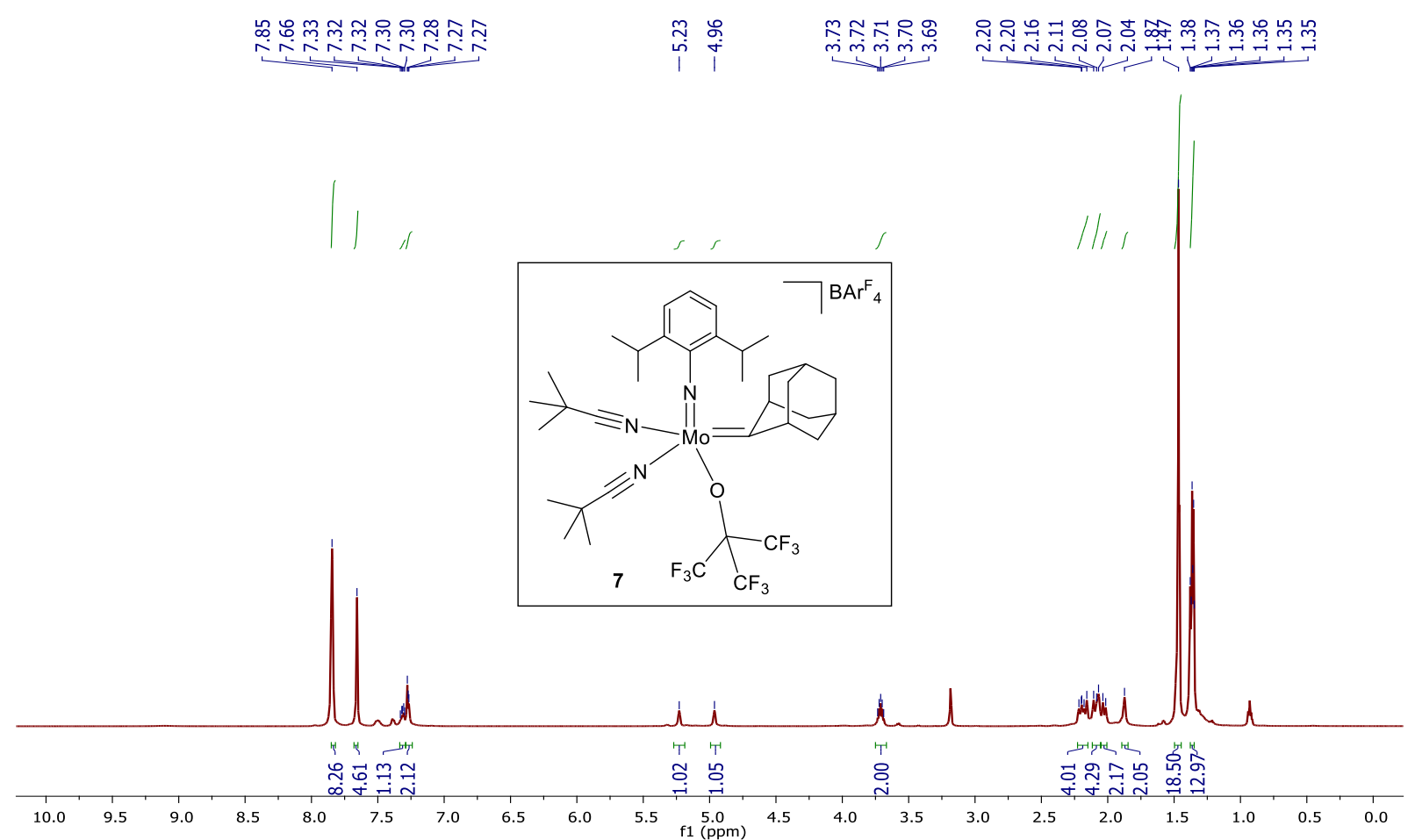

Figure S20. ${ }^{1} \mathrm{H}$ NMR spectrum of $\mathrm{Mo}(\mathrm{NAr})\left(\right.$ Adene) $\left(\mathrm{OC}_{4} \mathrm{~F}_{9}\right)(\mathrm{Piv})_{2}\left(\mathrm{BAr}_{4}\right)(7)$ recorded at $600 \mathrm{MHz}$ in $\mathrm{CD}_{2} \mathrm{Cl}_{2}$. 

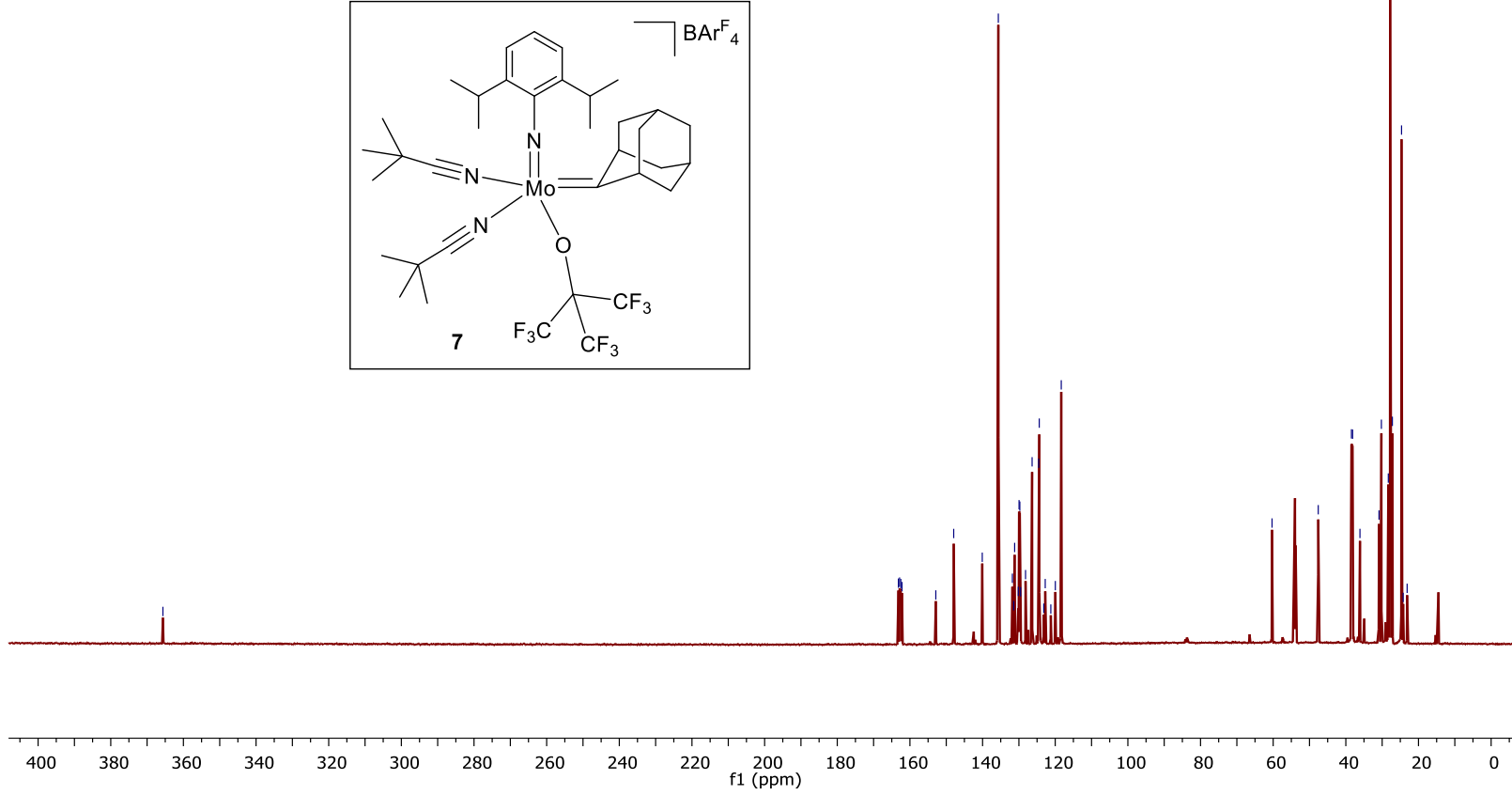

Figure S21. ${ }^{13} \mathrm{C}\left\{{ }^{1} \mathrm{H}\right\}$ NMR spectrum of $\mathrm{Mo}(\mathrm{NAr})($ Adene $)\left(\mathrm{OC}_{4} \mathrm{~F}_{9}\right)(\mathrm{Piv})_{2}\left(\mathrm{BAr}^{\mathrm{F}} 4\right)(7)$ recorded at 151 $\mathrm{MHz}$ in $\mathrm{CD}_{2} \mathrm{Cl}_{2}$.

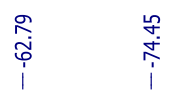
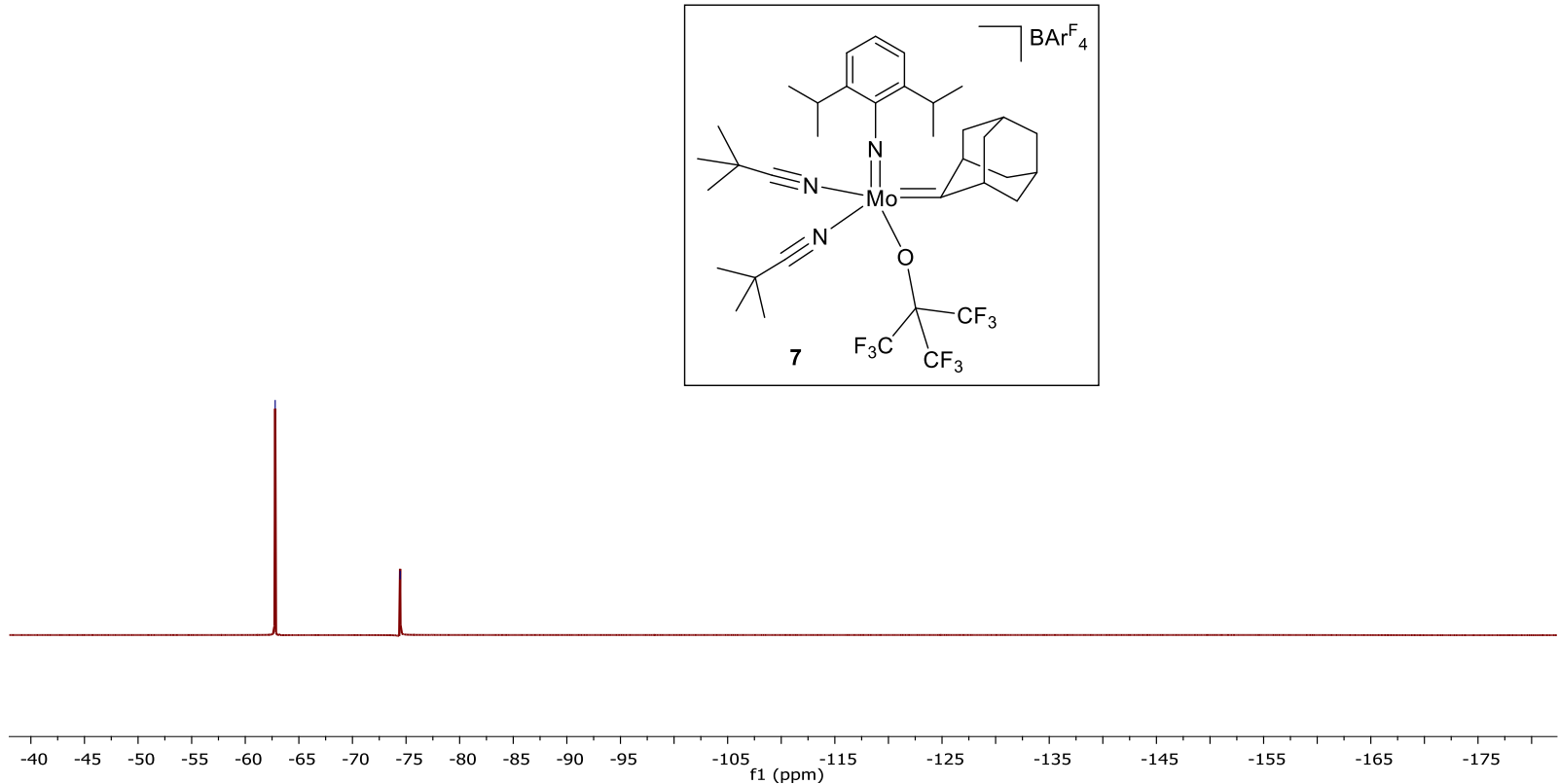

Figure S22. ${ }^{19} \mathrm{~F}\left\{{ }^{1} \mathrm{H}\right\}$ NMR spectrum of $\mathrm{Mo}(\mathrm{NAr})$ (Adene) $\left(\mathrm{OC}_{4} \mathrm{~F}_{9}\right)(\mathrm{Piv})_{2}\left(\mathrm{BAr}^{\mathrm{F}}{ }_{4}\right)(7)$ recorded at 564 $\mathrm{MHz}$ in $\mathrm{CD}_{2} \mathrm{Cl}_{2}$. 


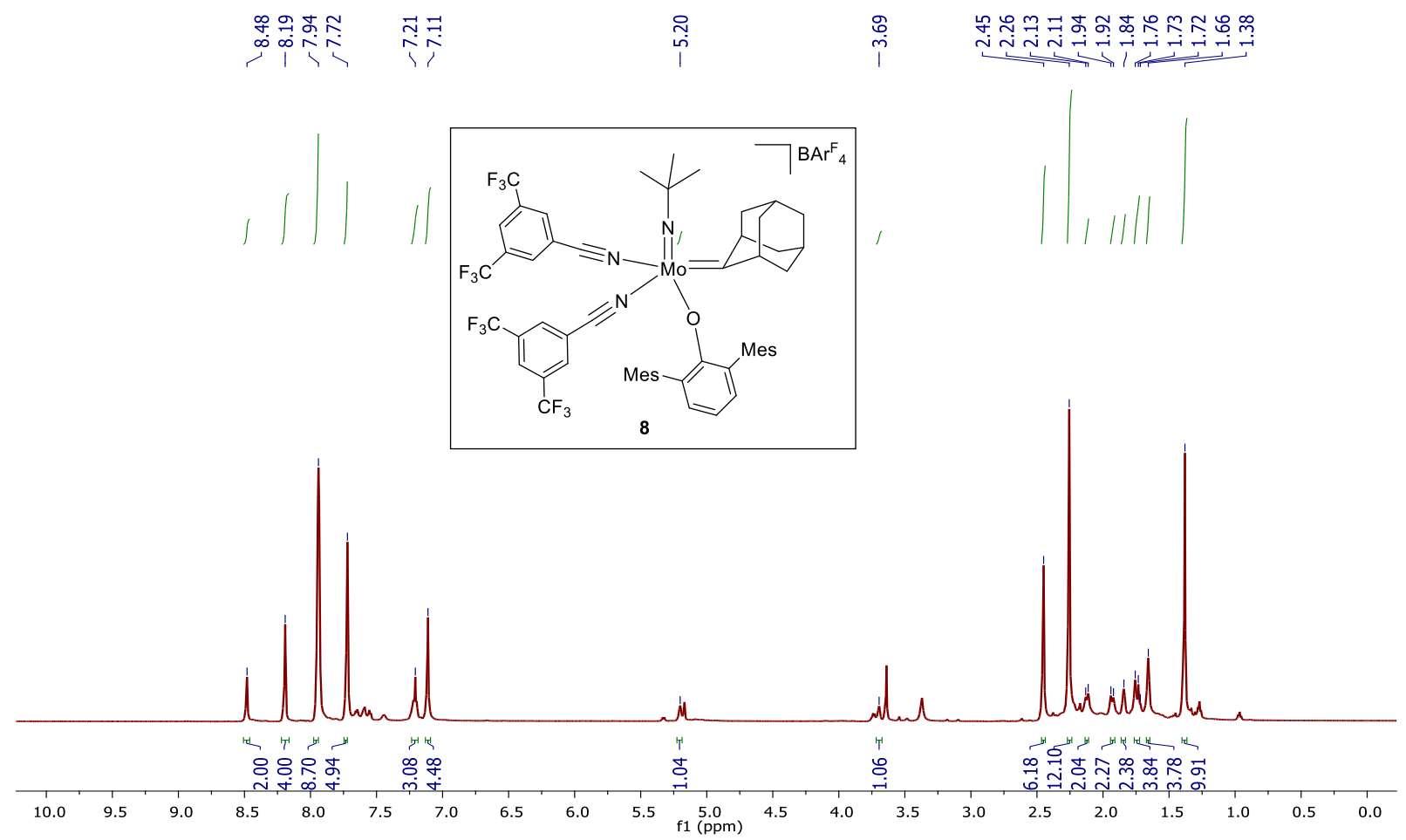

Figure S23. ${ }^{1} \mathrm{H}$ NMR spectrum of $\mathrm{Mo}(\mathrm{N}-t-\mathrm{Bu})\left(\right.$ Adene) $(\mathrm{OHMT})\left(\mathrm{Ph}^{\mathrm{F} 6} \mathrm{CN}\right)_{2}\left(\mathrm{BAr}^{\mathrm{F}} 4\right)(8)$ recorded at 600 $\mathrm{MHz}$ in $\mathrm{CD}_{2} \mathrm{Cl}_{2}$.

$\underset{\substack{\infty \\ i}}{\stackrel{\infty}{\infty}}$

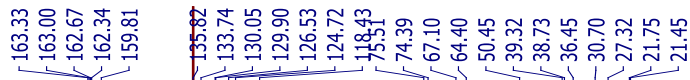
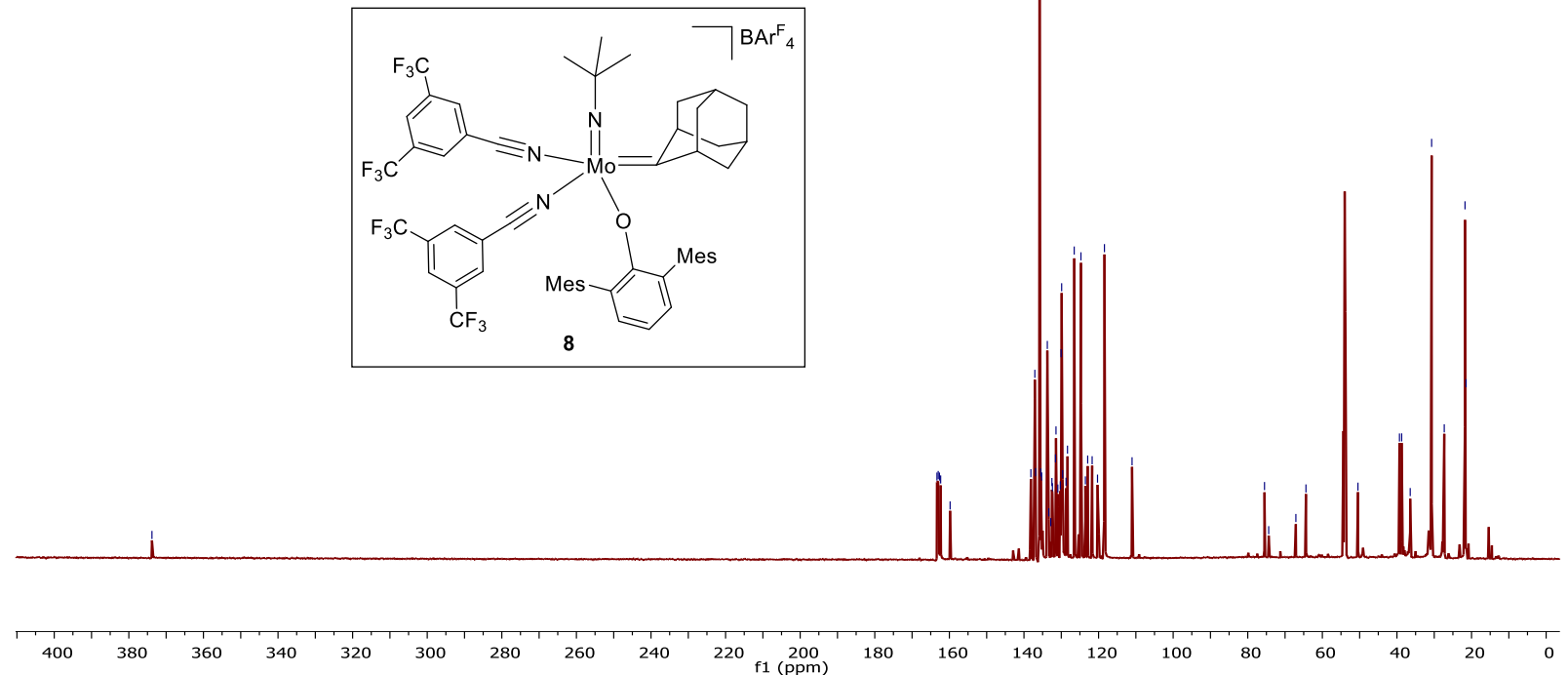

Figure S24. ${ }^{13} \mathrm{C}\left\{{ }^{1} \mathrm{H}\right\}$ NMR spectrum of $\mathrm{Mo}(\mathrm{N}-t-\mathrm{Bu})$ (Adene)(OHMT) $\left(\mathrm{Ph}^{\mathrm{F} 6} \mathrm{CN}\right)_{2}\left(\mathrm{BAr}^{\mathrm{F}} 4\right)(8)$ recorded at $151 \mathrm{MHz}$ in $\mathrm{CD}_{2} \mathrm{Cl}_{2}$. 


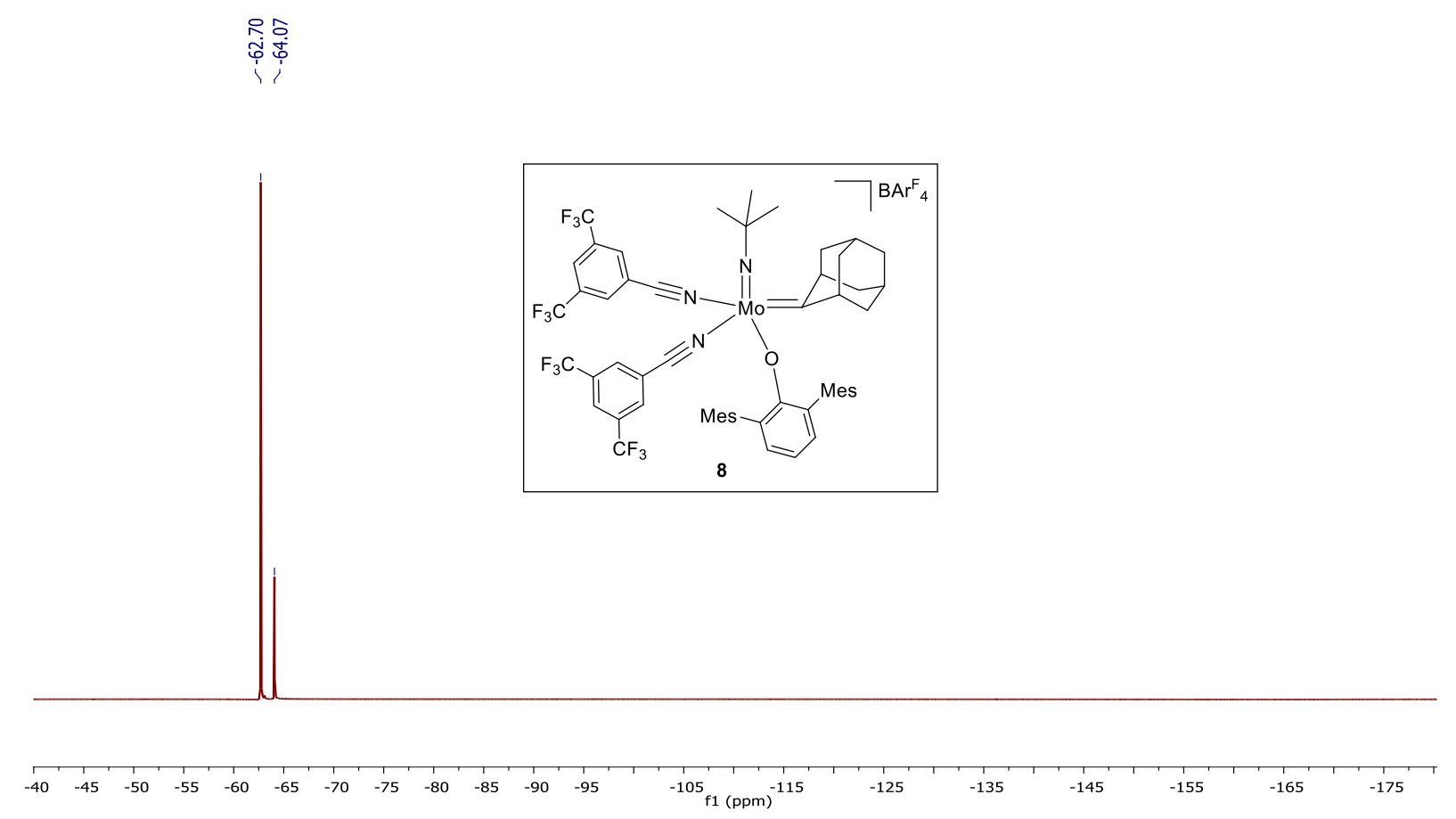

Figure S25. ${ }^{19} \mathrm{~F}\left\{{ }^{1} \mathrm{H}\right\}$ NMR spectrum of $\mathrm{Mo}(\mathrm{N}-t-\mathrm{Bu})($ Adene $)(\mathrm{OHMT})\left(\mathrm{Ph}^{\mathrm{F} 6} \mathrm{CN}\right)_{2}\left(\mathrm{BAr}^{\mathrm{F}} 4\right)(8)$ recorded at $564 \mathrm{MHz}$ in $\mathrm{CD}_{2} \mathrm{Cl}_{2}$.

\section{Catalytic Experiments}

\section{A1. Homo-Coupling of 1-Decene}

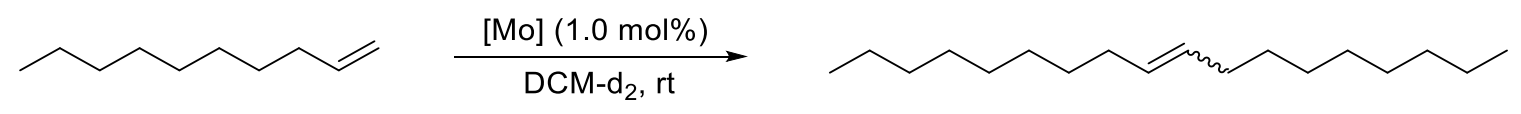

1-Decene (0.5 mmol) was added to a solution of Mo(VI)-complex (0.005 $\mathrm{mmol})$ in DCM- $\mathrm{d}_{2}(0.05 \mathrm{~mL})$ in an open vial. The reaction mixture was allowed to stir at $22{ }^{\circ} \mathrm{C}$ and aliquots were taken periodically and diluted with $0.5 \mathrm{~mL} \mathrm{CDCl}_{3}$ prior to collecting ${ }^{1} \mathrm{H} \mathrm{NMR}$ spectra. The conversion was determined by integration of the olefin proton resonance of 1decene $(\mathrm{m}, 5.86-5.78)$ and 9 -octadecene $(\mathrm{m}, 5.39-5.32)$. The $\mathrm{Z} / \mathrm{E}$ ratio was calculated through integration of the olefin proton resonance of E-9-octadecene (m, 5.39-5.37) and Z-9octadecene (m, 5.37-5.32).

Table S1. Conversion of 1-Decene to 9-Octadecene.

\begin{tabular}{|c|c|c|}
\hline [Mo] Complex & $\begin{array}{c}\text { Conversion } \\
(\%)\end{array}$ & $\begin{array}{c}\text { Selectivity } \\
\text { (Z/E) }\end{array}$ \\
\hline
\end{tabular}




\begin{tabular}{|c|c|c|}
\hline $\mathrm{Mo}\left(\mathrm{NC}_{6} \mathrm{~F}_{5}\right)($ Adene $)(\mathrm{OHMT})(\mathrm{Piv})_{2}\left(\mathrm{BAr}^{\mathrm{F}}{ }_{4}\right)(3)$ & 6 (3 days) & $50 / 50$ \\
\hline $\mathrm{Mo}\left(\mathrm{NC}_{6} \mathrm{~F}_{5}\right)($ Adene $)\left(\mathrm{OC}_{4} \mathrm{~F}_{9}\right)(\mathrm{Piv})_{2}\left(\mathrm{BArF}_{4}\right)(4)$ & 4 (3 days) & $33 / 66$ \\
\hline $\mathrm{Mo}\left(\mathrm{NC}_{6} \mathrm{~F}_{5}\right)($ Adene $)(\mathrm{OHMT})\left(\mathrm{Ph}^{\mathrm{F} 6} \mathrm{CN}\right)_{2}\left(\mathrm{BAr}_{4}\right)(5)$ & $\begin{array}{c}58(30 \mathrm{~min}) \\
66(24 \mathrm{~h}) \\
71(3 \text { days }) \\
87(1 \mathrm{~h})^{a} \\
91(24 \mathrm{~h})^{a}\end{array}$ & $\begin{array}{l}48 / 52 \\
\text { (3 days) } \\
35 / 65 \\
(24 \mathrm{~h})^{a}\end{array}$ \\
\hline $\mathrm{Mo}(\mathrm{NAr})$ (Adene) $(\mathrm{OHMT})\left(\mathrm{Ph}^{\mathrm{F} 6} \mathrm{CN}\right)_{2}\left(\mathrm{BAr}_{4}{ }_{4}\right)(6)$ & $\begin{array}{c}33(30 \mathrm{~min}) \\
34(2.5 \mathrm{~h}) \\
36(3 \text { days }) \\
56(1 \mathrm{~h})^{a} \\
61(24 \mathrm{~h})^{a}\end{array}$ & $\begin{array}{l}41 / 59 \\
\text { (3 days) } \\
37 / 63 \\
(24 \mathrm{~h})^{a}\end{array}$ \\
\hline $\mathrm{Mo}(\mathrm{NAr})($ Adene $)\left(\mathrm{OC}_{4} \mathrm{~F}_{9}\right)(\mathrm{Piv})_{2}\left(\mathrm{BAr}_{4} \mathrm{~F}_{4}\right)(7)$ & 4 (3 days) & $\begin{array}{c}25 / 75 \\
\text { (3 days) }\end{array}$ \\
\hline $\mathrm{Mo}(\mathrm{N}-t-\mathrm{Bu})($ Adene $)(\mathrm{OHMT})\left(\mathrm{Ph}^{\mathrm{F} 6} \mathrm{CN}\right)_{2}\left(\mathrm{BAr}_{4}{ }_{4}\right)(\mathbf{8})$ & $\begin{array}{l}7 \text { (30 min) } \\
11 \text { (3 days) }\end{array}$ & $\begin{array}{l}42 / 58 \\
\text { (3 days) }\end{array}$ \\
\hline
\end{tabular}

${ }^{a}$ With 2.5 mol\% catalyst.

\section{A2. Effect of $B\left(C_{6} F_{5}\right)_{3}$ in the Homocoupling of 1-Decene.}
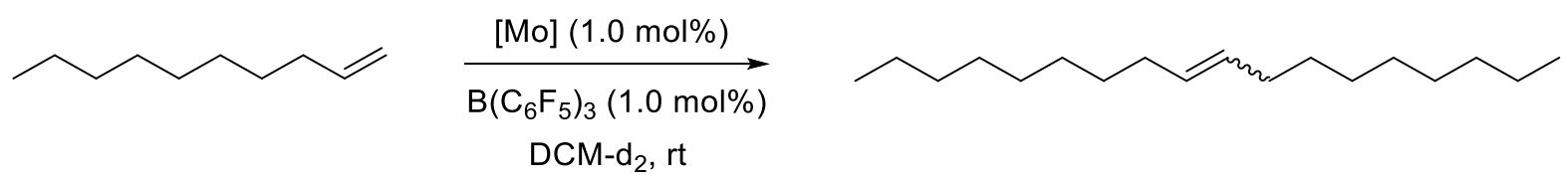

1-Decene (0.5 mmol) was added to a solution of Mo(VI)-complex $(0.005 \mathrm{mmol})$ and $\mathrm{B}\left(\mathrm{C}_{6} \mathrm{~F}_{5}\right)_{3}(0.005 \mathrm{mmol})$ in $\mathrm{DCM}-\mathrm{d}_{2}(0.05 \mathrm{~mL})$ in an open vial. The reaction mixture was allowed to stir at $22^{\circ} \mathrm{C}$ and aliquots were taken periodically and diluted with $0.5 \mathrm{~mL} \mathrm{CDCl}_{3}$ prior to collecting ${ }^{1} \mathrm{H}$ NMR spectra. The conversion was determined by integration of the olefin proton resonance of 1 -decene $(\mathrm{m}, 5.86-5.78)$ and 9-octadecene $(\mathrm{m}, 5.39-5.32)$. The Z/E 
ratio was calculated through integration of the olefin proton resonance of E-9-octadecene (m, 5.39-5.37) and Z-9-octadecene (m, 5.37-5.32).

Table S2. Effect of Lewis Acid $B\left(\mathrm{C}_{6} \mathrm{~F}_{5}\right)_{3}$ in the Conversion of 1-Decene to 9-Octadecene.

\begin{tabular}{|c|c|c|}
\hline Mo Complex & $\begin{array}{c}\text { Conversion } \\
(\%)\end{array}$ & $\begin{array}{c}\text { Selectivity } \\
\text { (Z/E) }\end{array}$ \\
\hline $\mathrm{Mo}\left(\mathrm{NC}_{6} \mathrm{~F}_{5}\right)($ Adene $)(\mathrm{OHMT})(\mathrm{Piv})_{2}\left(\mathrm{BAr}_{4}\right)(3)$ & $\begin{array}{l}95(1 \mathrm{~h}) \\
97(24 \mathrm{~h})\end{array}$ & $\begin{array}{l}21 / 79 \\
(24 \mathrm{~h})\end{array}$ \\
\hline $\mathrm{Mo}\left(\mathrm{NC}_{6} \mathrm{~F}_{5}\right)($ Adene $)\left(\mathrm{OC}_{4} \mathrm{~F}_{9}\right)(\mathrm{Piv})_{2}\left(\mathrm{BAr}_{4}\right)(4)$ & $\begin{array}{l}98(1 \mathrm{~h}) \\
99(24 \mathrm{~h})\end{array}$ & $\begin{array}{l}20 / 80 \\
(24 \mathrm{~h})\end{array}$ \\
\hline $\mathrm{Mo}\left(\mathrm{NC}_{6} \mathrm{~F}_{5}\right)($ Adene $)(\mathrm{OHMT})\left(\mathrm{Ph}^{\mathrm{F} 6} \mathrm{CN}\right)_{2}\left(\mathrm{BAr}_{4}{ }^{\mathrm{F}}\right)(5)$ & $\begin{array}{l}68(1 \mathrm{~h}) \\
84(24 \mathrm{~h})\end{array}$ & $\begin{array}{l}19 / 81 \\
(24 \mathrm{~h})\end{array}$ \\
\hline $\mathrm{Mo}(\mathrm{NAr})\left(\right.$ Adene)(OHMT) $\left(\mathrm{Ph}^{\mathrm{F} 6} \mathrm{CN}\right)_{2}\left(\mathrm{BAr}^{\mathrm{F}}{ }_{4}\right)(\mathbf{6})$ & $\begin{array}{c}96(1 \mathrm{~h}) \\
>99(24 \mathrm{~h})\end{array}$ & $\begin{array}{l}21 / 79 \\
(24 \mathrm{~h})\end{array}$ \\
\hline $\mathrm{Mo}(\mathrm{NAr})($ Adene $)\left(\mathrm{OC}_{4} \mathrm{~F}_{9}\right)(\mathrm{Piv})_{2}\left(\mathrm{BAr}_{4} \mathrm{~F}_{4}\right)(7)$ & $\begin{array}{c}90(1 \mathrm{~h}) \\
>99(24 \mathrm{~h})\end{array}$ & $\begin{array}{l}20 / 80 \\
(24 \mathrm{~h})\end{array}$ \\
\hline $\mathrm{Mo}(\mathrm{N}-t-\mathrm{Bu})($ Adene $)(\mathrm{OHMT})\left(\mathrm{Ph}^{\mathrm{F}}{ }^{\mathrm{C}} \mathrm{CN}\right)_{2}\left(\mathrm{BAr}_{4}{ }_{4}\right)(\mathbf{8})$ & $\begin{array}{l}92(1 \mathrm{~h}) \\
97(24 \mathrm{~h})\end{array}$ & $\begin{array}{l}20 / 80 \\
(24 \mathrm{~h})\end{array}$ \\
\hline
\end{tabular}

\section{B1. ROMP of Cyclooctene}
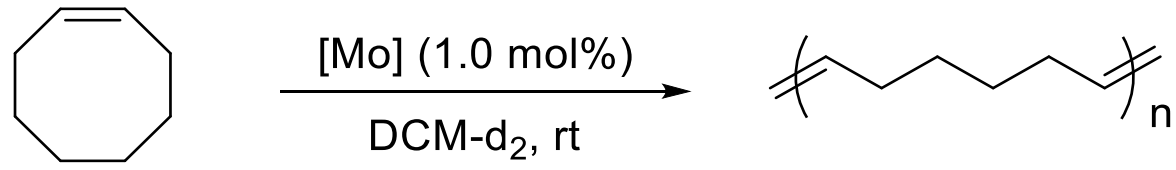

Cyclooctene (0.5 mmol) was added to a solution of the Mo(VI)-complex (0.005 mmol) in DCM- $\mathrm{d}_{2}(0.05 \mathrm{~mL})$ and the reaction mixture was stirred at $22{ }^{\circ} \mathrm{C}$. Aliquots were taken and diluted with $0.5 \mathrm{~mL} \mathrm{CDCl}_{3}$ prior to collecting ${ }^{1} \mathrm{H}$ NMR spectra. The conversion was calculated through integration of the olefin proton resonance of cyclooctene (m, 5.67-5.60 ppm) and poly-cyclooctene (m, 5.40-5.32 ppm). 
Table S3. ROMP of Cyclooctene

\begin{tabular}{|c|c|}
\hline Mo(VI)-Complex & $\begin{array}{c}\text { Conversion } \\
\text { (\%) }\end{array}$ \\
\hline $\mathrm{Mo}\left(\mathrm{NC}_{6} \mathrm{~F}_{5}\right)($ Adene $)(\mathrm{OHMT})(\mathrm{Piv})_{2}\left(\mathrm{BAr}_{4}\right)(3)$ & $\begin{array}{c}40(15 \mathrm{mins}) \\
55(2.5 \mathrm{~h}) \\
>99(24 \mathrm{~h})\end{array}$ \\
\hline $\mathrm{Mo}\left(\mathrm{NC}_{6} \mathrm{~F}_{5}\right)($ Adene $)\left(\mathrm{OC}_{4} \mathrm{~F}_{9}\right)(\mathrm{Piv})_{2}\left(\mathrm{BAr}_{4}\right)(4)$ & $\begin{array}{c}49(15 \mathrm{mins}) \\
67(2.5 \mathrm{~h}) \\
>99(24 \mathrm{~h})\end{array}$ \\
\hline $\mathrm{Mo}\left(\mathrm{NC}_{6} \mathrm{~F}_{5}\right)($ Adene $)(\mathrm{OHMT})\left(\mathrm{Ph}^{\mathrm{F} 6} \mathrm{CN}\right)_{2}\left(\mathrm{BAr}_{4}{ }_{4}\right)(5)$ & $>99$ (15 mins) \\
\hline $\mathrm{Mo}(\mathrm{NAr})($ Adene $)(\mathrm{OHMT})\left(\mathrm{Ph}^{\mathrm{F} 6} \mathrm{CN}\right)_{2}\left(\mathrm{BAr}_{4}\right)(\mathbf{6})$ & $\begin{array}{c}81(15 \mathrm{mins}) \\
>99(2.5 \mathrm{~h})\end{array}$ \\
\hline $\mathrm{Mo}(\mathrm{NAr})($ Adene $)\left(\mathrm{OC}_{4} \mathrm{~F}_{9}\right)(\mathrm{Piv})_{2}\left(\mathrm{BAr}_{4}\right)(7)$ & $\begin{array}{c}24 \text { (15 mins) } \\
40(2.5 \mathrm{~h}) \\
95(24 \mathrm{~h})\end{array}$ \\
\hline $\mathrm{Mo}(\mathrm{N}-t-\mathrm{Bu})($ Adene $)(\mathrm{OHMT})\left(\mathrm{Ph}^{\mathrm{F} 6} \mathrm{CN}\right)_{2}\left(\mathrm{BAr}^{\mathrm{F}} 4\right)(\mathbf{8})$ & 96 (15 mins) \\
\hline
\end{tabular}

\section{B2. Effect of $B\left(C_{6} F_{5}\right)_{3}$ in the ROMP of Cyclooctene}

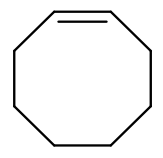

$$
\begin{gathered}
\underset{\mathrm{BMo}](1.0 \mathrm{~mol} \%)}{\mathrm{B}\left(\mathrm{C}_{6} \mathrm{~F}_{5}\right)_{3}(1.0 \mathrm{~mol} \%)} \\
\text { DCM-d } \mathrm{d}_{2}, \mathrm{rt}
\end{gathered}
$$<smiles>CCCCCCCCCC(C)(C)C</smiles>

Cyclooctene (0.5 mmol) was added to a solution of the Mo(VI)-complex (0.005 mmol) and $\mathrm{B}\left(\mathrm{C}_{6} \mathrm{~F}_{5}\right)_{3}(0.005 \mathrm{mmol})$ in $\mathrm{DCM}-\mathrm{d}_{2}(0.05 \mathrm{~mL})$ and the reaction mixture was stirred for $5-$ 15 minutes at $22{ }^{\circ} \mathrm{C}$. Aliquots were taken and diluted with $0.5 \mathrm{~mL} \mathrm{CDCl}_{3}$ prior to collecting ${ }^{1} \mathrm{H}$ NMR spectra. The conversion was calculated through integration of the olefin proton resonance of cyclooctene (m, 5.67-5.60 ppm) and poly-cyclooctene (m, 5.40-5.32 ppm). 
Table S4. Effect of Lewis Acid B $\left(\mathrm{C}_{6} \mathrm{~F}_{5}\right)_{3}$ in the ROMP of Cycloocetene.

\begin{tabular}{|c|c|}
\hline Mo(VI)-Complex & Conversion \\
(\%)
\end{tabular}

${ }^{a}$ With $0.1 \mathrm{~mol} \%$ catalyst; ${ }^{b}$ With $0.01 \mathrm{~mol} \%$ catalyst.

\section{C1. ROMP of 2,3-Dicarbomethoxynorbornadiene}<smiles>COC(OC)C1=C(C(OC)OC)C2C=CC1C2</smiles><smiles>CCCCCCC(C)C</smiles><smiles>C=CC1CC(C=C)C(C(=O)OC)=C1C(=O)OC</smiles>

A solution of Mo(VI)-complex ( $0.005 \mathrm{mmol})$ in DCM- $\mathrm{d}_{2}(0.5 \mathrm{~mL})$ was taken in a vial and then 2,3-dicarbomethoxynorbornadiene $(0.5 \mathrm{mmol})$ was added. After that the reaction mixture was allowed to stir for 15 minutes at $22^{\circ} \mathrm{C}$. Then, aliquot was taken and diluted with $0.5 \mathrm{~mL} \mathrm{CDCl} 3$ prior to collecting ${ }^{1} \mathrm{H}$ and ${ }^{13} \mathrm{C}$ NMR spectra. The conversion, cis-/transselectivity and tacticity (iso/syndio) was calculated from these spectra, following the literature procedure. ${ }^{1}$ 
Table S5. Polymerisation of 2,3-Dicarbomethoxynorbornadiene.

\begin{tabular}{|c|c|c|c|}
\hline Mo(VI)-Complex & $\begin{array}{c}\text { Conversion } \\
\text { (\%) }\end{array}$ & $\begin{array}{l}\text { Cis } \\
(\%)\end{array}$ & Iso/syndio \\
\hline $\mathrm{Mo}\left(\mathrm{NC}_{6} \mathrm{~F}_{5}\right)($ Adene $)(\mathrm{OHMT})(\mathrm{Piv})_{2}\left(\mathrm{BAr}_{4}\right)(3)$ & $\begin{array}{c}>99 \\
(15 \text { mins })\end{array}$ & 99 & $\begin{array}{c}16 / 84 \\
\text { (15 mins) }\end{array}$ \\
\hline $\mathrm{Mo}\left(\mathrm{NC}_{6} \mathrm{~F}_{5}\right)($ Adene $)\left(\mathrm{OC}_{4} \mathrm{~F}_{9}\right)(\mathrm{Piv})_{2}\left(\mathrm{BAr}_{4}\right)(4)$ & $\begin{array}{c}19(15 \mathrm{~min}) \\
25(5 \mathrm{~h}) \\
28(24 \mathrm{~h})\end{array}$ & 84 & $\begin{array}{l}32 / 68 \\
(24 \mathrm{~h})\end{array}$ \\
\hline $\mathrm{Mo}\left(\mathrm{NC}_{6} \mathrm{~F}_{5}\right)($ Adene $)(\mathrm{OHMT})\left(\mathrm{Ph}^{\mathrm{F} 6} \mathrm{CN}\right)_{2}\left(\mathrm{BAr}_{4}{ }_{4}\right)(5)$ & $\begin{array}{c}>99 \\
(15 \mathrm{~min})\end{array}$ & 99 & $\begin{array}{c}10 / 90 \\
(15 \text { mins })\end{array}$ \\
\hline $\mathrm{Mo}(\mathrm{NAr})($ Adene $\left.)(\mathrm{OHMT})\left(\mathrm{Ph}^{\mathrm{F} 6} \mathrm{CN}\right)_{2}\left(\mathrm{BAr}_{4}\right)^{\mathrm{F}}\right)(6)$ & $\begin{array}{l}\text { n.d. }(15 \mathrm{~min}) \\
\text { n.d. }(5 \mathrm{~h}) \\
\text { n.d. }(24 \mathrm{~h})\end{array}$ & - & - \\
\hline Mo(NAr)(Adene) $\left(\mathrm{OC}_{4} \mathrm{~F}_{9}\right)(\mathrm{Piv})_{2}\left(\mathrm{BAr}_{4}{ }_{4}\right)(7)$ & $\begin{array}{l}31(5 \mathrm{~h}) \\
83(24 \mathrm{~h})\end{array}$ & 90 & $\begin{array}{l}87 / 13 \\
(24 \mathrm{~h})\end{array}$ \\
\hline $\mathrm{Mo}(\mathrm{N}-t-\mathrm{Bu})($ Adene $)(\mathrm{OHMT})\left(\mathrm{Ph}^{\mathrm{F} 6} \mathrm{CN}\right)_{2}\left(\mathrm{BAr}^{\mathrm{F}}{ }_{4}\right)(\mathbf{8})$ & $84(3 \mathrm{~h})$ & 84 & $\begin{array}{l}16 / 84 \\
(3 \mathrm{~h})\end{array}$ \\
\hline
\end{tabular}

\section{C2. Effect of $B\left(C_{6} F_{5}\right)_{3}$ in the ROMP of 2,3-Dicarbomethoxynorbornadiene}<smiles>COC(=O)C1C2C=CC(C2)C1C(=O)OC</smiles>

$$
\frac{[\mathrm{Mo}](1.0 \mathrm{~mol} \%)}{\stackrel{\mathrm{B}\left(\mathrm{C}_{6} \mathrm{~F}_{5}\right)_{3}(1.0 \mathrm{~mol} \%)}{\mathrm{DCM}-\mathrm{d}_{2}, \mathrm{rt}}}
$$

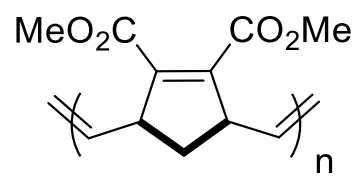

A solution of $\mathrm{Mo}(\mathrm{VI})$-complex $(0.005 \mathrm{mmol})$ and $\mathrm{B}\left(\mathrm{C}_{6} \mathrm{~F}_{5}\right)_{3}(0.005 \mathrm{mmol})$ in DCM- $\mathrm{d}_{2}$ $(0.5 \mathrm{~mL})$ were taken in a vial and then 2,3-dicarbomethoxynorbornadiene $(0.5 \mathrm{mmol})$ was added. After that the reaction mixture was allowed to stir for 15 minutes at $22{ }^{\circ} \mathrm{C}$. Then, aliquot was taken and diluted with $0.5 \mathrm{~mL} \mathrm{CDCl}_{3}$ prior to collecting ${ }^{1} \mathrm{H}$ and ${ }^{13} \mathrm{C} \mathrm{NMR}$ spectra. 
The conversion, cis-/trans- selectivity and tacticity (iso/syndio) was calculated from these spectra, following the literature procedure. ${ }^{1}$

Table S6. Effect of Lewis Acid B $\left(\mathrm{C}_{6} \mathrm{~F}_{5}\right)_{3}$ in the ROMP of 2,3-Dicarbomethoxynorbornadiene.

\begin{tabular}{|c|c|c|c|}
\hline Mo(VI)-Complex & $\begin{array}{c}\text { Conversion } \\
\text { (\%) }\end{array}$ & $\begin{array}{l}\text { Cis } \\
(\%)\end{array}$ & Iso/syndio \\
\hline $\mathrm{Mo}\left(\mathrm{NC}_{6} \mathrm{~F}_{5}\right)($ Adene $)(\mathrm{OHMT})(\mathrm{Piv})_{2}\left(\mathrm{BAr}_{4}\right)(3)$ & $\begin{array}{c}>99 \\
(5 \mathrm{~min})\end{array}$ & 99 & $16 / 84$ \\
\hline $\mathrm{Mo}\left(\mathrm{NC}_{6} \mathrm{~F}_{5}\right)($ Adene $)\left(\mathrm{OC}_{4} \mathrm{~F}_{9}\right)(\mathrm{Piv})_{2}\left(\mathrm{BAr}_{4}\right)(4)$ & $24(1 \mathrm{~h})$ & 84 & $15 / 85$ \\
\hline $\mathrm{Mo}\left(\mathrm{NC}_{6} \mathrm{~F}_{5}\right)($ Adene $)(\mathrm{OHMT})\left(\mathrm{Ph}^{\mathrm{F} 6} \mathrm{CN}\right)_{2}\left(\mathrm{BAr}_{4}{ }_{4}\right)(5)$ & $\begin{array}{c}>99 \\
(5 \mathrm{~min})\end{array}$ & 99 & $03 / 97$ \\
\hline $\mathrm{Mo}(\mathrm{NAr})($ Adene $)(\mathrm{OHMT})\left(\mathrm{Ph}^{\mathrm{F} 6} \mathrm{CN}\right)_{2}\left(\mathrm{BAr}_{4}{ }^{\mathrm{F}}\right)(6)$ & n.d. $(1 \mathrm{~h})$ & - & - \\
\hline $\mathrm{Mo}(\mathrm{NAr})($ Adene $)\left(\mathrm{OC}_{4} \mathrm{~F}_{9}\right)(\mathrm{Piv})_{2}\left(\mathrm{BAr}_{4}\right)(7)$ & n.d. (1 h) & - & - \\
\hline $\mathrm{Mo}(\mathrm{N}-t-\mathrm{Bu})($ Adene $)(\mathrm{OHMT})\left(\mathrm{Ph}^{\mathrm{F} 6} \mathrm{CN}\right)_{2}\left(\mathrm{BAr}^{\mathrm{F}}{ }_{4}\right)(\mathbf{8})$ & $27(1 \mathrm{~h})$ & 27 & $09 / 91$ \\
\hline
\end{tabular}

\section{X-Ray Crystallography}

Diffraction data were collected on a Bruker D8 Venture Duo diffractometer with a Bruker Photon III CPAD detector using Mo $K_{\alpha}$ radiation $(\lambda=0.71073 \AA$ ) from an I $\mu \mathrm{S}$ microsource. Data were collected at $100 \mathrm{~K}$ by performing $0.5^{\circ} \phi$ - and $\omega$-scans, integrated using SAINT $^{2}$, and scaled using SADABS ${ }^{3}$. The structure was solved by dual-space methods using SHELXT $^{4}$ and refined against $F^{2}$ on all data by full-matrix least squares with SHELXL-2018/35 following established refinement strategies ${ }^{6}$. All non-hydrogen atoms were refined anisotropically, and all hydrogen atoms were included into the model at geometrically calculated positions and refined using a riding model. The isotropic displacement parameters of all hydrogen atoms were fixed to 1.2 times the $U$ value of the atoms they are linked to (1.5 times for methyl groups). Crystal and data quality details, as well as a summary of the residual refinement values, are listed in the accompanying table. 


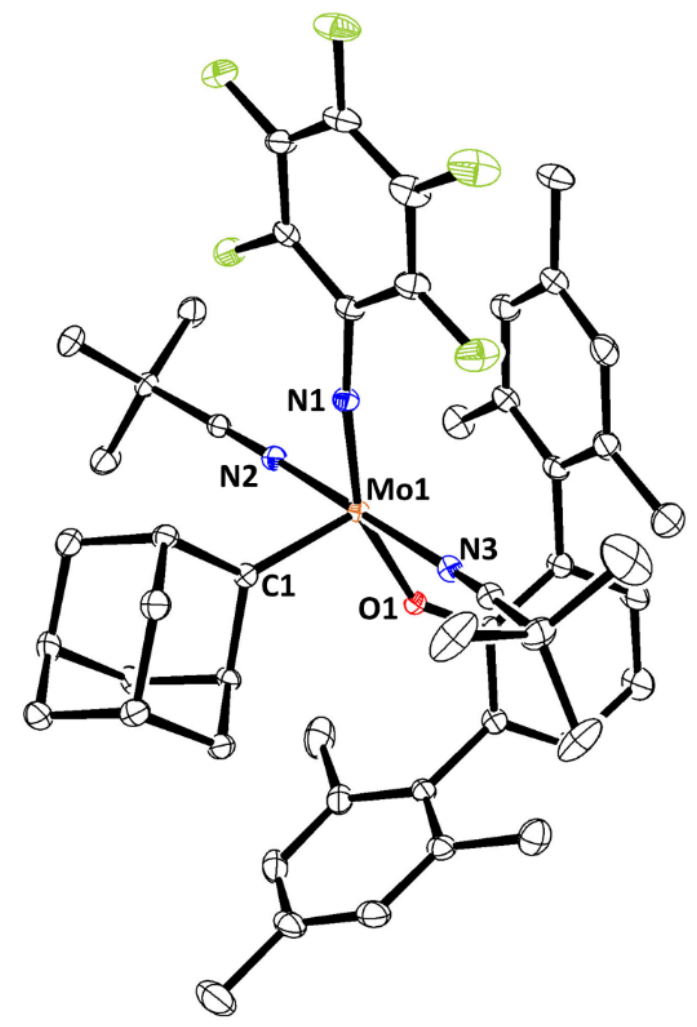

Figure S26. Thermal ellipsoid plot (30\%) of $\mathrm{Mo}\left(\mathrm{NC}_{6} \mathrm{~F}_{5}\right)\left(\right.$ Adene) $(\mathrm{OHMT})\left(\mathrm{Piv}_{2}\left(\mathrm{BAr}_{4}\right)^{\mathrm{F}}\right)$. Solvent molecule pentane, $\mathrm{BAr}^{\mathrm{F}} 4$ anion and hydrogen atoms have been omitted for clarity. Selected bond length (Å): Mo1-C1, 1.913(3); Mo1-N1, 1.758(3); Mo1-N2, 2.163(3); Mo1-N3, 2.152(3); Mo1-01, 1.931(2).

Compound $\mathrm{Mo}\left(\mathrm{NC}_{6} \mathrm{~F}_{5}\right)\left(\right.$ Adene) $(\mathrm{OHMT})(\mathrm{Piv})_{2}\left(\mathrm{BAr}_{4}\right)$ crystallizes in the centrosymmetric monoclinic space group $P 2_{1} / c$ with two molecules of cationic $\mathrm{Mo}\left(\mathrm{NC}_{6} \mathrm{~F}_{5}\right)$ (Adene)(OHMT)(Piv) ${ }_{2}\left(\mathrm{BAr}_{4}\right)$, two molecules of anionic BArF, and two molecules of pentane per asymmetric unit. The crystals were of only moderate quality, and the structure should be considered accordingly. One molybdenum-bound pivalonitrile ligand and five $\mathrm{BAr}^{\mathrm{F}}$ trifluoromethyl groups exhibit positional disorder that were each modeled over two positions with freely refined disorder ratios. All disorders was refined with the help of similarity restraints on 1,2- and 1,3- distances as well as similarity and rigid bond restraints for anisotropic displacement parameters. The anisotropic displacement parameters for the two nearly-overlapping nitrogen positions of the disordered pivalonitrile ligand were constrained to be equivalent and restrained to be approximately isotropic. 
Table S7. Crystal Data and Structure Refinement for $\mathrm{Mo}\left(\mathrm{NC}_{6} \mathrm{~F}_{5}\right)$ (Adene)(OHMT)(Piv) $)_{2}\left(\mathrm{BAr}_{4}{ }_{4}\right)$.

Identification code

Empirical formula

Formula weight

Temperature

Wavelength

Crystal system

Space group

Unit cell dimensions

Volume

$\mathrm{Z}$

Density (calculated)

Absorption coefficient

$\mathrm{F}(000)$

Crystal color

Crystal size

Theta range for data collection

Index ranges

Reflections collected

Independent reflections

Completeness to theta $=25.242^{\circ}$

Absorption correction

Refinement method

Data / restraints / parameters

Goodness-of-fit on $\mathrm{F}^{2}$ rrs69bp

C87 H81 B F29 Mo N3 O

1842.29

$100(2) \mathrm{K}$

$0.71073 \AA$

Monoclinic

$\mathrm{P} 21 / \mathrm{c}$

$\mathrm{a}=25.0638(14) \AA \quad \alpha=90^{\circ}$

$\mathrm{b}=15.9111(9) \AA$

$\beta=90.6045(16)^{\circ}$

$c=42.587(2) \AA$

16982.3(16) $\AA^{3}$

8

$1.441 \mathrm{Mg} / \mathrm{m}^{3}$

$0.266 \mathrm{~mm}^{-1}$

7520

yellow

$0.230 \times 0.100 \times 0.084 \mathrm{~mm}^{3}$

1.261 to $29.130^{\circ}$

$-34<=h<=34,-21<=k<=21,-58<=l<=58$

380624

$45713[\mathrm{R}(\mathrm{int})=0.1028]$

$100.0 \%$

Semi-empirical from equivalents

Full-matrix least-squares on $\mathrm{F}^{2}$

45713 / 649 / 2442

1.141

Final R indices [I>2sigma(I) $=36335$ data] $\mathrm{R} 1=0.0797, \mathrm{wR} 2=0.1691$

R indices (all data, $0.73 \AA$ )

$\mathrm{R} 1=0.1020, \mathrm{wR} 2=0.1794$

Largest diff. peak and hole

1.698 and -1.392 e. $\AA^{-3}$ 


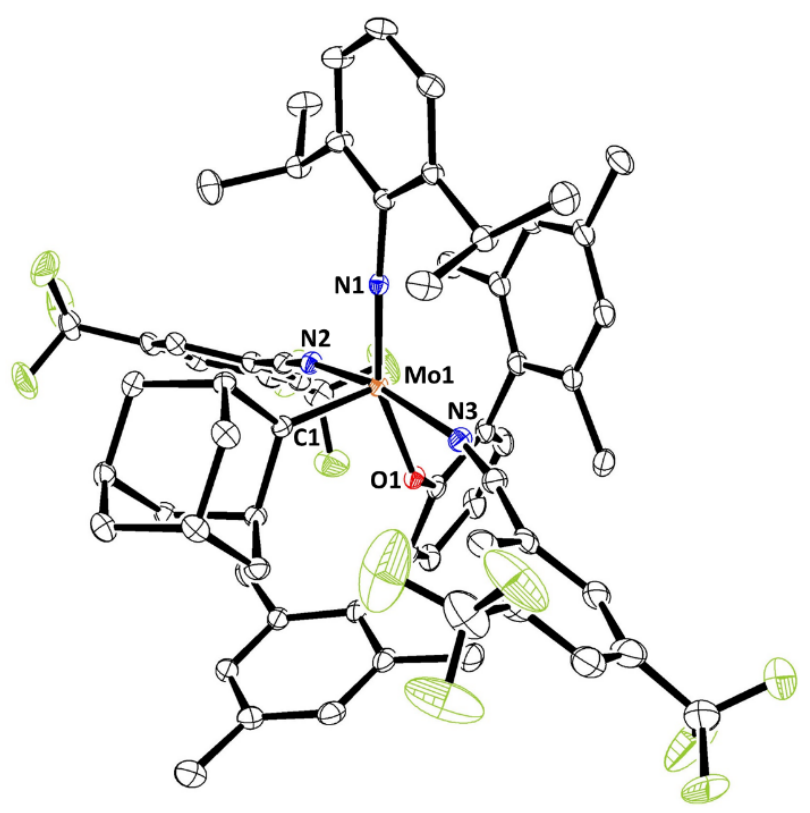

Figure S27. Thermal ellipsoid plot (30\%) of $\mathrm{Mo}(\mathrm{NAr})\left(\right.$ Adene)(OHMT) $\left(\mathrm{Ph}^{\mathrm{F} 6} \mathrm{CN}\right)_{2}\left(\mathrm{BAr}^{\mathrm{F}} 4\right)$. Solvent molecule pentane, $\mathrm{BAr}_{4}{ }_{4}$ anion and hydrogen atoms have been omitted for clarity. Selected bond length (Å): Mo1-C1, 1.9216(15); Mo1-N1, 1.7524(13); Mo1-N2, 2.1405(13); Mo1-N3, 2.1431(14); Mo1-01, 1.9712(11).

Compound $\mathrm{Mo}(\mathrm{NAr})($ Adene $)(\mathrm{OHMT})\left(\mathrm{Ph}^{\mathrm{F} 6} \mathrm{CN}\right)_{2}\left(\mathrm{BAr}_{4}^{\mathrm{F}}\right)$ crystallizes in the centrosymmetric triclinic space group $P-1$ with one molecule of cationic $\mathrm{Mo}(\mathrm{NAr})$ (Adene)(OHMT) $\left(\mathrm{Ph}^{\mathrm{F} 6} \mathrm{CN}\right)_{2}\left(\mathrm{BAr}_{4}{ }_{4}\right)$ and one molecule of anionic $\mathrm{BAr}^{\mathrm{F}}$ per asymmetric unit. The cationic molybdenum molecule contains one aryl group, one isopropyl group, and two trifluoromethyl groups that exhibit positional disorder, and the $\mathrm{BAr}^{\mathrm{F}}$ anion contains four additional trifluoromethyl groups that exhibit positional disorder; each of these disorders were modeled over two positions with freely-refined disorder ratios. All disorders was refined with the help of similarity restraints on 1,2- and 1,3- distances as well as similarity and rigid bond restraints for anisotropic displacement parameters. Flatness restraints were also used for the disordered aryl group. The anisotropic displacement parameters for two nearly-overlapping carbon positions of a disordered trifluoromethyl group were constrained to be equivalent. 
Table S8. Crystal Data and Structure Refinement for $\mathrm{Mo}(\mathrm{NAr})\left(\right.$ Adene)(OHMT) $\left(\mathrm{Ph}^{\mathrm{F} 6} \mathrm{CN}\right)_{2}\left(\mathrm{BAr}^{\mathrm{F}} 4\right)$.

Identification code

Empirical formula

Formula weight

Temperature

Wavelength

Crystal system

Space group

Unit cell dimensions

Volume

$\mathrm{Z}$

Density (calculated)

Absorption coefficient

$\mathrm{F}(000)$

Crystal color

Crystal size

Theta range for data collection

Index ranges

Reflections collected

Independent reflections

Completeness to theta $=25.242^{\circ}$

Absorption correction

Refinement method

Data / restraints / parameters

Goodness-of-fit on $\mathrm{F}^{2}$ rrs77bp

C101 H86 B F36 Mo N3 O

2148.47

100(2) K

$0.71073 \AA$

Triclinic

P'1

$$
\begin{array}{ll}
\mathrm{a}=14.1503(6) \AA & \alpha=71.5295(15)^{\circ} . \\
\mathrm{b}=18.7923(9) \AA & \beta=86.3103(15)^{\circ} . \\
\mathrm{c}=19.2718(8) \AA & \gamma=88.8389(16)^{\circ} .
\end{array}
$$$$
4850.6(4) \AA^{3}
$$

2

$1.471 \mathrm{Mg} / \mathrm{m}^{3}$

$0.254 \mathrm{~mm}^{-1}$

2184

yellow

$0.276 \times 0.093 \times 0.067 \mathrm{~mm}^{3}$

1.442 to $31.506^{\circ}$

$-20<=h<=20,-27<=k<=27,-28<=l<=28$

221205

32312 [R(int $)=0.0430]$

$100.0 \%$

Semi-empirical from equivalents

Full-matrix least-squares on $\mathrm{F}^{2}$

32312 / 3281 / 1650

1.068

Final $\mathrm{R}$ indices [I>2sigma(I) $=27773$ data]

$\mathrm{R} 1=0.0455, \mathrm{wR} 2=$

0.1128

R indices (all data, $0.68 \AA$ )

$\mathrm{R} 1=0.0553, \mathrm{wR} 2=0.1184$

Largest diff. peak and hole

1.048 and -0.835 e. $\AA^{-3}$ 


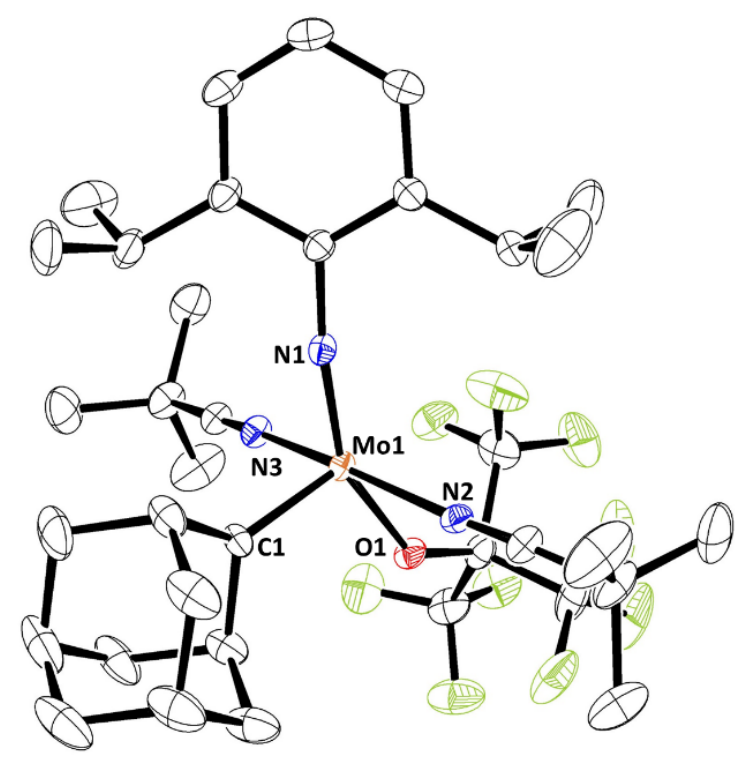

Figure S28. Thermal ellipsoid plot (30\%) of Mo(NAr)(Adene) $\left(\mathrm{OC}_{4} \mathrm{~F}_{9}\right)(\mathrm{Piv})_{2}\left(\mathrm{BAr}_{4}\right)$. Solvent molecule pentane, $\mathrm{BAr}_{4}{ }_{4}$ anion and hydrogen atoms have been omitted for clarity. Selected bond length (Å): Mo1-C1, 1.905(3); Mo1-N1, 1.7334(14); Mo1-N2, 2.144(3); Mo1-N3, 2.1446(16); Mo1-01, 2.019(2).

Compound $\mathrm{Mo}(\mathrm{NAr})($ Adene $)\left(\mathrm{OC}_{4} \mathrm{~F}_{9}\right)\left(\mathrm{Piv}_{2}\left(\mathrm{BAr}_{4}\right)\right.$ crystallizes in the centrosymmetric triclinic space group $P-1$ with one molecule of cationic $\mathrm{Mo}(\mathrm{NAr})$ (Adene) $\left(\mathrm{OC}_{4} \mathrm{~F}_{9}\right)(\mathrm{Piv})_{2}\left(\mathrm{BAr}_{4} \mathrm{~F}_{4}\right.$, one molecule of anionic $\mathrm{BAr}$, and one half of one molecule of pentane per asymmetric unit. The cationic molybdenum molecule contains one adamantylidene ligand, one isobutylnitrile ligand, and one perfluoro-t-butoxy ligand that exhibit positional disorder, and the $\mathrm{BAr}^{\mathrm{F}}$ anion contains six trifluoromethyl groups that exhibit rotational disorder; each of these disorders were modeled over two positions with freely-refined disorder ratios. All disorders was refined with the help of similarity restraints on 1,2- and 1,3- distances as well as similarity and rigid bond restraints for anisotropic displacement parameters. 
Table S9. Crystal data and structure refinement for $\mathrm{Mo}(\mathrm{NAr})(\mathrm{Adene})\left(\mathrm{OC}_{4} \mathrm{~F}_{9}\right)(\mathrm{Piv})_{2}\left(\mathrm{BAr}_{4}\right)$.

Identification code

Empirical formula

Formula weight

Temperature

Wavelength

Crystal system

Space group

Unit cell dimensions

Volume

$\mathrm{Z}$

Density (calculated)

Absorption coefficient

$\mathrm{F}(000)$

Crystal color

Crystal size

Theta range for data collection

Index ranges

Reflections collected

Independent reflections

Completeness to theta $=25.242^{\circ}$

Absorption correction

Refinement method

Data / restraints / parameters

Goodness-of-fit on F2 rrs75bp

C70.50 H67 B F33 Mo N3 O

1706.02

$180(2) \mathrm{K}$

$0.71073 \AA$

Triclinic

P $\overline{1}$

$\mathrm{a}=13.4991(8) \AA \quad \alpha=109.448(2)^{\circ}$.

$\mathrm{b}=14.4691(8) \AA \quad \beta=100.941(2)^{\circ}$.

$\mathrm{c}=21.4663(13) \AA \quad \gamma=95.816(2)^{\circ}$.

$3819.9(4) \AA 3$

2

$1.483 \mathrm{Mg} / \mathrm{m} 3$

$0.296 \mathrm{~mm}-1$

1726

yellow

$0.228 \times 0.169 \times 0.056 \mathrm{~mm} 3$

1.497 to $30.508^{\circ}$

$-19<=h<=19,-20<=k<=20,-30<=l<=30$

231906

$23350[\mathrm{R}$ (int $)=0.0578]$

$100.0 \%$

Semi-empirical from equivalents

Full-matrix least-squares on F2

23350 / 2027 / 1460

1.076

Final $\mathrm{R}$ indices [I>2sigma(I) = 19445 data $]$

$\mathrm{R} 1=0.0455, \quad \mathrm{wR} 2=$

0.1085

$\mathrm{R}$ indices (all data, $0.70 \AA \AA$ )

$\mathrm{R} 1=0.0589, \mathrm{wR} 2=0.1157$

Largest diff. peak and hole

0.752 and -0.807 e. $\AA \AA-3$ 


\section{References}

1. Forrest, W. P.; Axtell, J. C.; Schrock, R. R. Tungsten Oxo Alkylidene Complexes as Initiators for the Stereoregular Polymerization of 2,3Dicarbomethoxynorbornadiene. Organometallics 2014, 33, 2313-2325.

2. SAINT, version 8.40, Bruker (2019), Bruker AXS Inc., Madison, Wisconsin, USA.

3. SADABS, version 2016/2, Bruker (2016), Bruker AXS Inc., Madison, Wisconsin, USA.

4. Sheldrick, G. M., SHELXT -Integrated Space-Group and Crystalstructure Determination. Acta Cryst. 2015, A71, 3-8.

5. Sheldrick, G. M., Crystal Structure Refinement with SHELXL. Acta Cryst. 2015, C71, 38.

6. Müller, P., Practical Suggestions for Better Crystal Structures. Crystallogr. Rev. 2009, 15, 57-83. 